\title{
La conservation de la viande par l'auréomycine, en milieu tropical
}

\author{
par G. MEMERY et C. LABOUCHE
}

\section{INTRODUCTION}

Dès 1948, le Dr. Taar, directeur de la Section Expérimentale des Pêcheries de Vancouver (Canada) recherche, avec ses collaborateurs, un moyen d'enrayer efficacement le développement des bactéries en vue d'une meilleure conservation du poisson. Ils utilisent, dans ce but, un certain nombre d'antibiotiques, parmi lesquels l'auréomycine, la terramycine, le chloramphénicol donnent les résultats les plus probants, lauréomycine étant le plus efficace.

Les antibiotiques sont ensuite essayés avec plus ou moins de succès comme agents de conservation d'un grand nombre de denrécs périssables destinées à l'alimentation humaine. Goldberg et coll. (1954) en les mélangeant à du bouf haché maintiennent la qualité du produit pendant 5 à 9 jours, à $+10^{\circ} \mathrm{C}$.

Au Congrès de Los Angeles, cn juin 1954, l'efficacité d'une immersion de trente minutes, dans une solution d'antibiotique est unanimement reconnue pour la conservation de la volaille.

En 1949, Anderson et coll. emploient avec succès la subtilinc pour suppléer en partie à la stérilisation par la chaleur de certaines conserves fragiles.

Mais les expériences les plus intéressantes, bien que les moins nombreuses, sont celles portant sur les carcasses d'animaux de boucherie et plus particulièrement de bovins. Deatherage (1952) expérimente sur 7 sujets et obtient des résultats très encourageants. La conservation des carcasses serait prolongée de plusieurs jours et, bien que la viande traitée puisse parfois paraître " avancée ", la qualité serait parfaite et le goût inchangé. Le traitement consiste en la perfusion d'une solution d'auréomycine après saignés complète des animaux.

Ces conclusions sont confirmées, en 1954, par
Weiser et coll., qui constatent d'autre part une augmentation caractéristique de la tendreté de la viande traitée, lorsquelle est conservée à $20-24^{\circ} \mathrm{C}$.

Plus récemment (1957), Ginsberg et coll. recherchent au Kenya un mode d'emploi pratique de l'auréomycine pour la conservation de la viande sous climats tropicaux ou sub-tropicaux. Ils se placent dans les conditions locales de manipulation de la viande par les Africains, depuis l'abattage jusqu'à l'étal du boucher, et ils concluent à l'intérêt de l'auréomycine pour la conservation de la viande dans les pays chauds et sous-développés, bien qu'elle ne puisse en aucun cas remplacer le froid.

\section{RECHERCHES EFFECTUEES AU LABORATOIRE DE DAKAR}

\section{I. - BUTS DE L'EXPERIMENTATION}

L'expérimentation a quatre buts essentiels : - confirmer ou infirmer l'efficacité de l'auréomycine dans la conservation des carcasses de bovins en milieu tropical ;

- rechercher une méthode permettant de chiffrer l'efficacité de l'antibiotique sans laisser place à une interprétation subjective ;

- mettre au point une technique d'utilisation de l'antibiotique pouvant s'intercaler facilement entre les mancuvres d'abattage et les manipulations de carcasses ;

- entrevoir si l'application de ces techniques pourrait permettre de pallier, en pays tropicaux sous-développés, les défauts d'une chaîne de froid discontinue.

\section{II. - PROTOCOLE}

Six expériences se déroulent d'avril à décembre 1957. Une seule, celle' d'octobre. a 
lieu durant la saison des pluies, les autres se situent en périodes relativement plus sèches et moins chaudes.

Mis à part des facteurs climatiques, variables, elles sont toutes cffectuécs dans les mêmes conditions, dans l'enceinte des laboratoires. Les quelques variantes sont signalées à chaque fois.

Pour la clarté de l'exposé, nous divisons le déroulement des expériences en trois temps :

jer temps - abattage des animaux et traitement des carcasses.

$2^{\circ}$ temps - mode, lieu et durée de conservation des carcasses.

$3^{\text {e }}$ temps - prélèvements, examens et dosages permettant d'apprécier l'état de conservation ou de putréfaction des carcasses.

\section{Abattage des animaux.}

Locaux : les abattages ont lieu dans une salle entièrement carrelée, 'd'une propreté rigoureuse et largement éclairée et aérée par des fenêtres grillagées.

Un palan permet une manipulation facile des carcasses.

Les animaux choisis sont des bovins mâles, jeunes, castrés, de race locale (sans bosse et zébus, ou croisements des deux), d'âge variable. Nous nous sommes efforcés de réunir dans chaque lot des animaux sensiblement de même âge, de même race et de même poids. Leur état général est souvent mauvais, et les carcasses seraient classées pour la plupart dans la troisième qualité.

Notre expérimentation porte sur 17 bovins, d'un poids vif compris entre 85 et $150 \mathrm{~kg}$, gardés à l'étable depuis plusieurs semaines et abattus de ce fait dans les meilleures conditions de repos requises.

Abattage : Le sacrifice des animaux est effectué suivant la méthode locale, qui consiste en la section totale de la partie inférieure de l'encolure jusqu'aux vertèbres, au niveau de la troisième vertèbre cervicale.

L'éviscération et l'habillage des carcasses sont accomplis par du personnel africain, sous notre direction; les règles classiques d'hygiène sont toujours scrupuleusement respectées au cours des mancuvres.

La viande des animaux est généralement plus proche de la viande de veau que de la viande de bœuf. Et la diversité entre l'âge et l'état général des animaux d'un lot à l'autre peut être à l'origine de certaines variations dans les résultats.

\section{$2^{\circ}$ Traitement des carcasses.}

\section{A. - Produits UTILISES}

Nous avons utilisé l'auréomycine (1) sous trois formes :

- SDA 682 ou Acronix V à 2,45\% d'antibiotique pour la perfusion ;

- SDA 680 ou Acronix B à 10\% d'antibiotique pour la pulvérisation ;

- Chorhydrate d'auréomycine pur pour les injections intraveineuses.

Les solutions sont préparées extemporanément à partir de ces produits en poudre, conservés en chambre froide.

\section{B. - Methode De traitement}

\section{Traitement par perfusion.}

On injecte, sous pression modérée, pour obtenir une bonne diffusion, un liquide dans la carotide d'un animal venant d'être saigné.

La solution à perfuser est contenue dans un flacon en verre de 12 litres fermé par un bouchon en caoutchouc, à deux ouvertures, solidement fixé. Une canne de verre allant jusqu'au fond du flacon, continuée par un tube de caoutchouc, permet son injection par l'intermédiairc d'un trocart. Par l'autre ouverture est insufflé dans le flacon, au-dessus du liquide, de l'air comprimé sous pression réduite.

Les animaux sont d'abord saignés à blanc, selon la technique décrite, puis une carotide est isolée afin d'y fixer solidement le trocart à injection.

Les perfusions durent de 4 à 6 minutes sous une pression de 150 à 200 grammes par centiriètre carré, constamment vérifiée par un manomètre incorporé dans le détendeur.

40,8 grammes d'Acronix V sont dissous dans 6 litres d'eau distillée stérile, ce qui représente une concentration en auréomycine pure de 166 p.p.m. (2), soit un gramme pour 6 litres de solution.

(I) Fournie gracieusement par la Société Parisienne d'Expansion Chimique (SPECIA)

(2) p. p. m. : partie par million. 
Les injections sont faites sur la base de 6 litres de solution pour 100 kilogs de poids vif.

\section{Traitement par injections intraveineuses.}

On pratique unc injection intraveineuse à l'animal quelque temps avant son abattage.

Le matériel nécessaire consiste en une seringue de $100 \mathrm{~cm}^{3}$ et une aiguille intraveineuse pour gros animaux.

Aucune préparation particulière n'est requise. L'injection est pratiquée de 1 h. $1 / 2$ à 3 heures avant l'abattage; le moment optimum semble se situer aux environs de la $2^{\mathrm{e}}$ heure. Elle doit être poussée lentement pour éviter un choc toujours possible. L'animal choqué reste dans un état d'abattement et d'hébétude pendant 1 à 5 minutes puis reprend une attitude normale.

Un gramme de chlorydrate d'auréomycine est dissous dans $100 \mathrm{~cm}^{3}$ d'eau distillée. La solution obtenue est employée à raison de $100 \mathrm{~cm}^{3}$ pour $100 \mathrm{~kg}$ de poids vif.

\section{Traitement par pulvérisation.}

On pulvérise une solution d'auréomycine sur les carcasses, après habillage, au cours du ressuage.

La pulvérisation la meilleure et la plus uniforme est obtenue avec un pistolet à peinture branché sur une bouteille d'air comprimé, munie d'un détendeur.

Après l'habillage, les carcasses sont laissées, pendant une quinzaine de minutes, pendues, afin qu'elles subissent un commencement de séchage.

Une solution d'Acronix B à 2,5 grammes par litre est utilisée, ce qui représente une concentration en auréomycine de 250 p.p.m. soit 25 centigrammes d'auréomycine pure par litre. Chaque carcasse reçoit $1 / 2$ litre de cette sclution, soigneusement répartie sur toute la surface y compris les endroits les moins accessibles.

\section{Choix d'une méthode.}

La perfusion est critiquable. Elle est cependant utilisée comme test de référence, étant donné les résultats obtenus par d'autres expérimentateurs. Une expérience préalable, faite avec une solution coloré au trypan bleu, montre que, pour obtenir une bonne perfusion, il ne faut pas injecter une quantité de liquide inférieure ’̀ 6 litres pour 100 kilogrammes de poids vif. Or cette pratique, à notre avis, peut être assimilée à une fraude. Les carcasses sont en effet souvent pesées avant ressuage complet, c'est-à-dire avant que toute l'eau soit éliminée et les pertes de poids qu'elles subiront par la suite seront supéricures à celles normalement prévues $\left(^{*}\right)$ par l'acheteur. Cependant au point de vue de la qualité, nous n'avons pas remarqué de différence de tenue importante entre les carcasses perfusées et les carcasses témoins.

Dans son application, cette méthode entraîne un certain nombre de difficultés. Elle ralentit sensiblement les manipulations d'abattage en retardant l'éviscération. Elle nécessite un matériel assez encombrant ou fragile, tel que bouteille $\mathrm{d}$ 'cir comprimé, flacons de verre de 10 à 15 litres. Elle demande une certaine habileté pour isoler la carotide après section de l'encolure et pour fixer d'une façon convenable le trocart, afin d'éviter une fuite ou une rupture qui entrainerait une perte de liquide importante et une mauvaise perfusion.

Les avantages de l'injection intraveineuse, par rapporì à la précédente, sont certains. Elle n'entrave en rien les manipulations des carcasses, n'entraîne aucun apport de liquide après la mort de l'animal, ne nécessite aucun appareillage encombrant. L'injection, qui doit être faite deux heures avant l'abattage, ne présente pas de difficultés, les animaux restant souvent stationnés un temps beaucoup plus long dans les enclos des abattoirs avant l'abattage. La mise au repos du bétail avant sacrifice est d'ailleurs prescrit par les règles d'hygiène. La pratique de l'injection intraveineuse est facile chez les grands animaux si la contention est bien faite. Bien que certains sujets puissent être choqués lors de l'injection, l'abattement passager qui en résulte ne laisse aucune trace. Nous n'avons jamais noté d'accidents durables et ce procédé permet même une manipulation plus facile des animaux.

La pulvérisation des carcasses que nous avons employée, soit seule, soit en association avec les deux techniques précédentes, est aussi d'une application aisée. Elle s'effectue en cours de ressuage et n'entrave en rien les manipulations d'abattoir. Etant donné la forte concentration (250 p.p.m.) d'auréomycine de la solution, une légère couleur jaune peut apparaître au niveau des aponévroses et des tendons des carcasses.

La conduite de notre expérimentation est la suivante :

- chaque lot est formé généralement de trois animaux : l'un ne subit aucun traitement spécial

(*) de 1 à 2 p. 100. 
et sert de témoin ; un autre est traité par perfusion suivie d'une pulvérisation, le troisième reçoit une injection intraveineuse avant abattage et la carcasse est ensuite pulvérisée. Les carcasses ne sont pas l'objet de soins particuliers qui ne puissent être effectués dans unabattoir normal.

\section{$3^{\circ}$ Conservation des carcasses.}

Les carcasses sont entreposées pendant la durée de chaque expérience dans la salle même où a lieu l'abattage : salle propre et bien aérée. Une chambre froide, réglée entre $+2^{\circ}$ et $4^{\circ} \mathrm{C}$, sert à l'entreposage de trois demi-carcasses en vue d'un essai de conservation sous antibiotique et froid.

Les carcasses sont suspendues par un palonnier à une potence individuelle. Elles n'ont aucun contact entre elles et ne touchent par aucun point ni au mur ni au sol. Leur disposition, par rapport aux fenêtres et à la porte d'entrée, est identique, c'est-à-dire que les conditions d'aération, de température ct d'hygrométric sont toujours les mêmes pour les trois carcasses étudiées simultanément.

Elles sont soigneusement enveloppées pendant la durée de chaque expérience avec du tissu "moustiquaire " pour les protéger des mouches. Cette protection est complétée par une pulvérisation quotidienne d'insecticide. D'autre part des moisissures s'étant développées rapidement à la surface de la viande traitée, nous avons essayé d'inhiber leur prolifération par une pulvérisation d'acide sorbique. Ce produit s'est avéré inefficace.

La durée de conservation des carcasses varie suivant les lots. Elle est pour le témoin de 4 jours au minimum et de 7 jours au maximum ; généralement nous avons été obligés de nous débarrasser de ce demier avant les autres carcasses bien qu'aucun examen complémentaire n'ait été effectué. Le lot mis en chambre froide est resté 22 jours entre $+2^{\circ}$ et $+4^{\circ}$ (avant d'être replacé à la température ordinaire pendant 5 jours).

La salle d'entreposage, largement aérée et peu isolée, est soumise aux variations de température et d'hygrométrie extérieures, qui sont enregistrées au cours de chaque expérience à proximité immédiate du lot de carcasses.

\section{$4^{\circ}$ Appréciation de l'état de conservation des carcasses.}

Il est procédé à un examen toutes les 24 heures, un essai préalable ayant montré que, plus fréquent, il ne donne pas de meilleurs résultats.

Il comporte :

un examen de l'état général,

un examen organoleptique,

un examen bactériologique,

une analyse chimique et une mesure de $\mathrm{jH}$.

\section{EXAMEN DE L'ÉTAT GÉNÉRAL}

On note l'aspect général, l'apparition et la disparition de la rigidité cadavérique, l'existence ou l'absence d'écoulement en dessous de chaque carcasse.

\section{EXAMEN ORGANOLEPTIQUe}

La viande, après chaque prélèvement, est examinée sur la coupe fraîche. Sa couleur, son odeur, son suintement sont notés, ainsi que la présence ou l'absence de gaz lors de putréfaction débutante.

\section{EXAMEN BACTÉRIOLOGIQUE}

Il porte sur trois points précis :

- numération de germes totaux par gramme de viande, effectuée seulement sur les carcasses des 3 premiers lots ;

- étude succincte comparative de la flore rencontrée sur les carcasses traitées et non traitées.

- recherche des souches auréomycine-résistantes.

\section{Numération des germes totaux.}

Les prélèvements sont toujours effectués de façon stérile, dans la même masse musculaire, après qu'ait été enlevée une couche de viande de $2 \mathrm{~cm}$ d'épaisseur. Chacun d'eux est constitué de 4 petits morceaux, prélevés en profondeur en quatre points différents de la coupe, avec un scalpel et une pince stériles, et introduits audessus de la flamme dans le tube du microbroyeur Durel et Sausse. Les tubes ramenés au laboratoire sont pesés pour permettre de calculer le poids exact de viande. Dans chaque tube, $5 \mathrm{~cm}^{3}$ d'eau physiologique par gramme de viande sont ajoutés puis le tout est broyé, toujours stérilement.

A partir du broyat obtenu, des dilutions décimales en bouillon sont opérées, en changeant de pipette à chaque dilution.

Parallèlement, une numération sur plaque de 
gélose en boîte de Pétri est effectuée à partir d'un centimètre cube des dilutions précédentes.

Les lectures sont faites après 48 heures d'étuve à $37^{\circ} \mathrm{C}$.

Etude de la flore.

La flore végétant sur chaque carcasse est très soigneusement étudiée. Les identifications de germes, souvent longues ct délicates, ne sont pas poussées au delà d'une discrimination grossière, suffisante cependant pour permettre une comparaison entre la flore de deux carcasses différentes.

\section{Recherche de la résistance à l'auréomycine}

Un test de résistance à l'auréomycine, par la méthode du disque sur plaques de gélose, est effectué sur tous les germes isolés à partir des carcasses, dans le but de rechercher des souches résistantes pouvant se manifester.

\section{Analyse chimiQue et pH}

Les analyses chimiques consistent essentiellement en des dosages de l'azote basique volatil total de la viande. La putréfaction de la viande, quelle que soit sa forme, les enzymes mis en jeu et les microorganismes aérobies ou anaérobies en cause, entraînent toujours l'apparition, parmi d'autres produits de dégradation, de corps contenant tous de l'azote et groupés sous le nom d'azote basique volatil, dont l'ammoniac est le terme le plus simple. Nous avons pensé pouvoir suivre l'évolution de la maturation puis de la putréfaction de la viande en dosant ces produits.

Deux méthodes sont utilisées :

- la distillation sur appareil de Schloesing et Aubin et le microdosage de Conway.

\section{Méthode de Schloesing et Aubin.}

Nous ne décrirons pas cette méthode très classique, mais nous signalerons les modifications apportées par nous dans son application.

L'extraction aqueuse de chaque échantillon porte sur 100 gr de viande fraîche broyée au mixer. Une défécation est faite à l'acide trichloracétique, à raison de $70 \mathrm{~cm}^{3}$ d'une solution à 25 p. 100, par extraction. Après filtration, l'extrait obtenu est ajusté à $1.000 \mathrm{~cm}^{3}$.

Les prises d'essai sont de $250 \mathrm{~cm}^{3}$, nous remplaçons la magnésie par du carbonate de lithium plus soluble.

Cette méthode que nous avons utilisée au début de notre expérimentation est longue, ne permet que peu de dosages à la fois, demande un matériel encombrant et semble manquer de précision pour de faibles valeurs en azote basiq volatil, même en opérant avec des solutions $\mathrm{d}$ 'acide sulfurique et de soude $\mathrm{N} / 50$.

D'autre part, lors des distillations, l'acide trichloracétique ayant servi à la défécation est décomposé et forme du chloroforme, qui vient se condenser dans le distillat, ce qui nous oblige ensuite à le déplacer par chauffage, car son action sur l'indicateur coloré rend le virage plus sensible lors de la titrimétrie.

\section{Microdosage de Conway.}

Après des dosages comparatifs nombreux, la méthode d'extraction est modifiée et rendue plus rapide tout en restant aussi efficace. Le temps d'agitation est supprimé, tandis que l'extraction et la défécation sont faites simultanément au cours du broyage au mixer. D'autre part le volume de l'extrait est ramené de $1.000 \mathrm{~cm}^{3}$ à $500 \mathrm{~cm}^{3}$.

Dans cette méthode, l'azote basique volatil est déplacé par du carbonate de potassium en solution aqueuse saturée et vient se fixer sur de l'acide borique à 1 p. 100 . Le dosage s'effectue avec la microburette de Conway, avec de l'acide chlorhydrique $\mathrm{N} / 50$ qui ramène le $\mathrm{pH}$ de la solution d'acide borique à sa valeur initiale.

Les avantages de cette méthode résident dans sa rapidité d'exécution, la possibilité qu'elle offre de faire un grand nombre de dosages simultanés et, par suite, d'exécuter plusieurs dosages à partir d'un même extrait, enfin sa sensibilité aux faibles concentrations d'azote basique volatil.

La mesure du $\mathrm{pH}$ de la viande est pratiquée suivant la méthode du Professeur Drieux (1944), consistant à faire macérer $10 \mathrm{~g}$. de viande sans graisse, ni sang, ni tissu conjonctif, pendant 30 minutes dans 5 à 10 fois son volume d'eau distillée fraîchement bouillie et refroidie. A $5 \mathrm{~cm}^{3}$ de la macération on ajoute 10 gouttes d'indicateur coloré.

Tous les examens n'ont pas donné de renseignements exploitables. C'est ainsi que nous avons dû abandonner la mesure du $\mathrm{pH}$ à partir du cinquième lot et la numération des germes totaux à partir du quatrième. En effet, les valeurs du $\mathrm{pH}$ obtenues ne nous ont jamais permis de faire de déduction valable, soit que les $\mathrm{pH}$ ini- 
tiaux des viandes soient variables, soit que leur évolution soit contradictoire. Quant à la numération des germes totaux elle ne répond pas au but recherché; il n'y a pas de corrélation étroite entre le nombre de germes par gramme de viande et son état de décomposition. L'étude de la flore est en effet assez variable d'une carcasse à l'autre, bien qu'elles soient toutes traitées dans des conditions identiques. Or, il semble que la qualité des germes présents a plus d'incidence sur le degré de putréfaction que leur nombre absolu. Des germes pcu ou pas protéolytiques tels que le colibacille et germes apparentés, les paracoli, certains staphylocoques, etc. peuvent se développer en grand nombre sans créer de dégradation importante de la viande et sans pour autant la rendre dangereuse ; par contre d'autres germes, même en petit nombre, sont capables de provoquer une putréfaction rapide grâce à leur pouvoir protéolytique et putréfiant, tels les germes des genres $P$ seu- domonas, Proteus et Serratia, certains bacillus, et surtout les Clostridium.

Les dosages chimiques sont menés en deux temps par la méthode de Schloesing et Aubin pour les lots I et II, par la micro-méthode de Conway pour les autres. Le résultat final exprimé est la moyenne arithmétique des résultats de trois dosages différents à partir du même extrait. Une étude statistique de ces résultats fait l'objet d'un chapitre particulier. Les prélèvements de viande, comme nous l'avons déjà indiqué, sont toujours effectués dans la même masse musculaire, en l'occurrence le tende de tranche.

Nous devons signaler que nous avons voulu doser la triméthylamine par la méthode de Conway; mais ce corps existe en trop faible quantité dans la viande, fût-elle en état de putréfaction avancée, pour que son dosage soit de quelque intérêt.

\section{III. - RÉSULTATS}

Ils sont présentés sous deux rubriques :

A. - Tableaux résumant chaque expérience, et commentaires.

B. - Tableaux groupant les dosages de l'azote basique volatil, et l'étude statistique.

\section{A. - TABLEAUX DES EXPÉRIENCES et COMMENTAIRES}

\section{LEGENDE : abréviations}

$\mathrm{N}$ : numéro des animaux

$\mathrm{C}:$ catégoric : $\mathrm{p}:$ pulvérisé

Pp : perfusé et pulvérisé

Ivp : traité en intraveineuse et pulvérisé

$\mathrm{T}$ : témoin

Temps : 24, 72, etc.

date des prélèvements $24,72,96$ heures après abattage.

germes totaux : nombre total par gramme de viande. 
azote basique : azote basique volatil total en milligrammes par kilogramme de viande.

Même signification pour tous les tableaux.

\section{Température - Hygrométrie}

Max : moyenne des maxima enregistrés pendant la durée de l'expérience.

Min : moyenne des minima enregistrés pendant la durée de l'expérience.

Max Ext. : Valeur maxima la plus élevée enregistrée pendant la durée de l'expérience.

Min Ext. : Valeur minima la plus faible enregistrée pendant la durée de l'expérience.

LOT n० 1 - 24 Avril - 29 Avril 1957

TABLEAD I

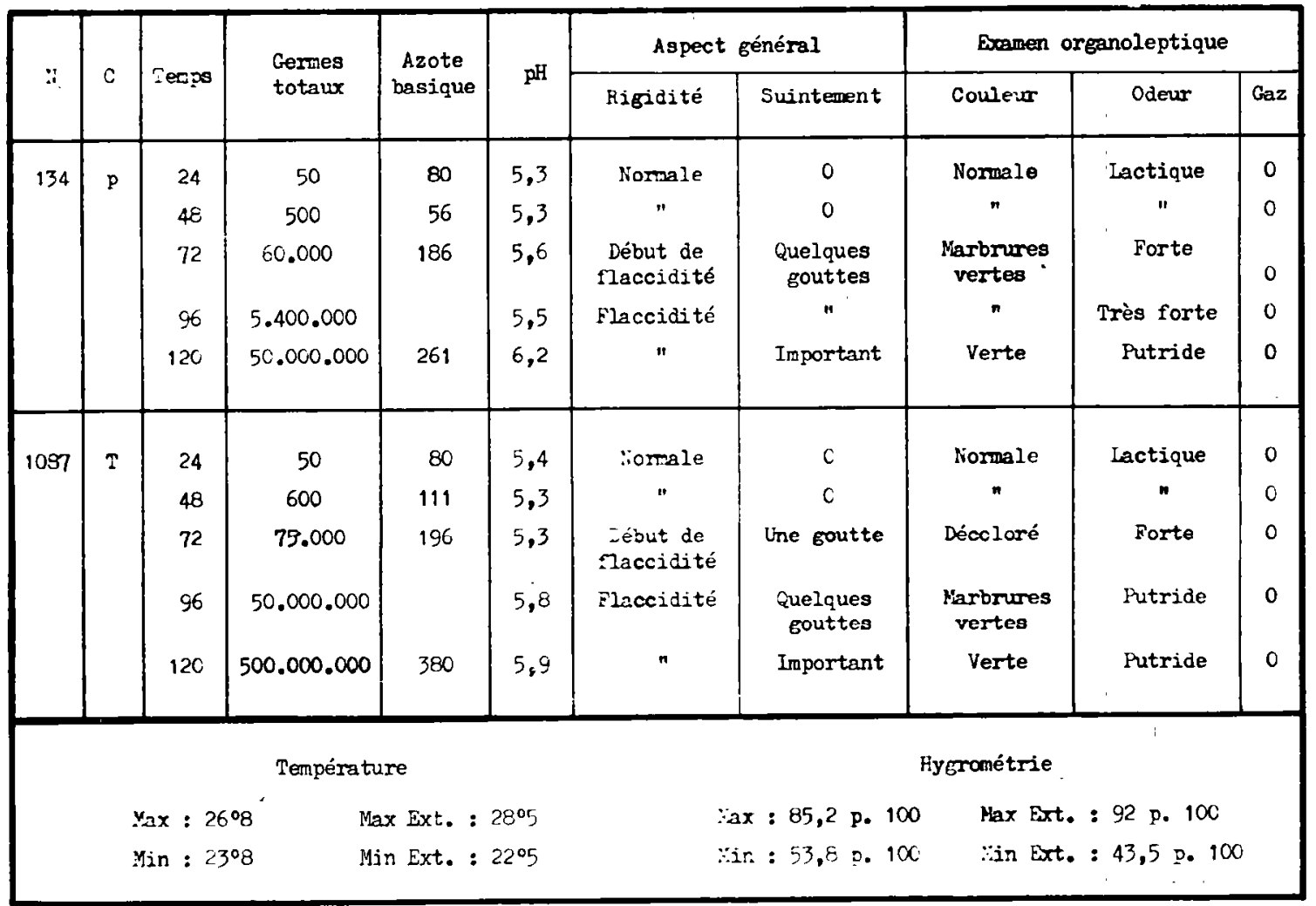

Nous ne constatons aucune différence essentielle entre les résultats de la carcasse témoin et ceux de la carcasse pulvérisée. Le dosage de l'azote volatil total de la $96^{\text {e }}$ heure n'a pu être mené à bien du fait d'un incident de distillation. 


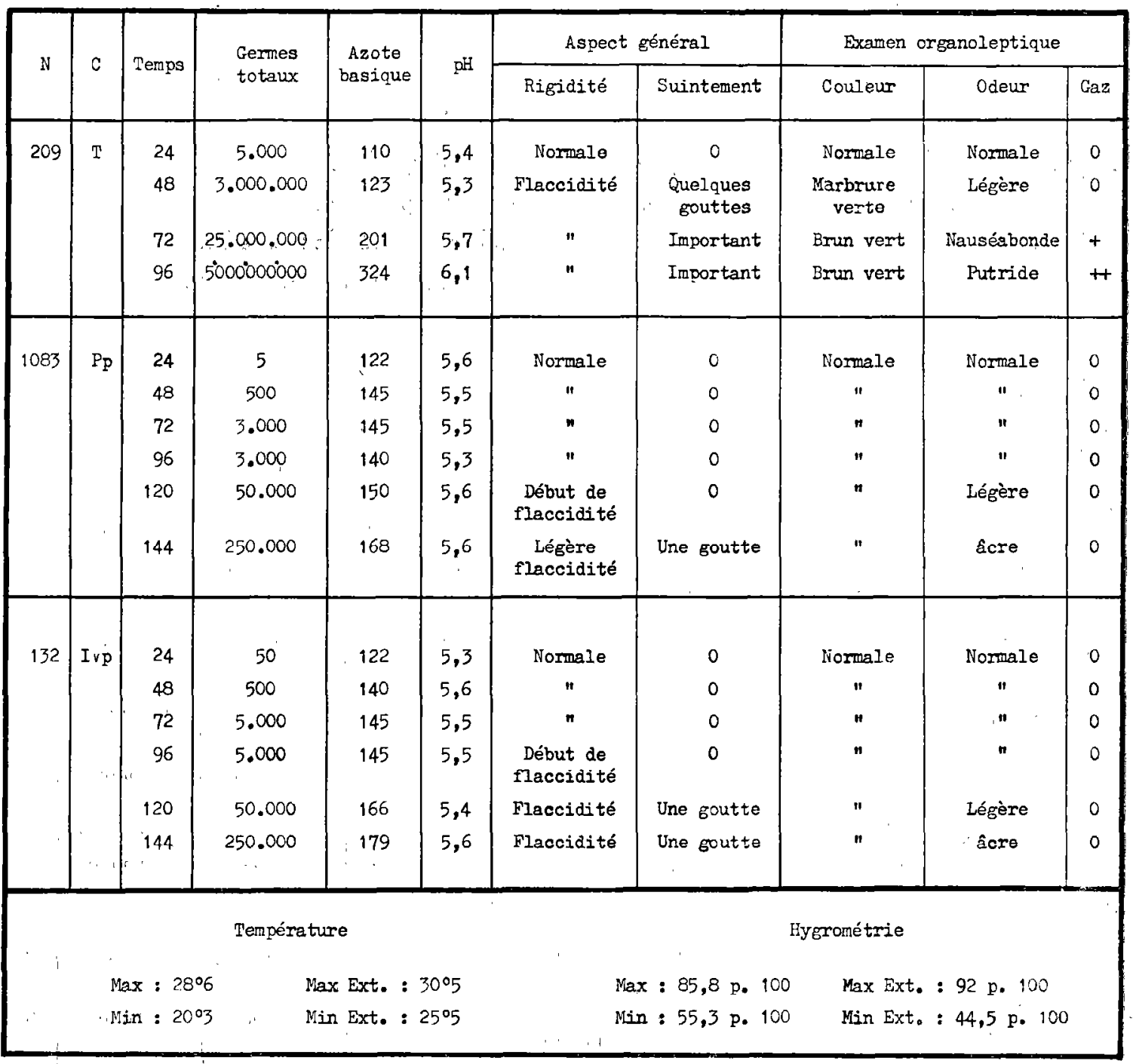

Les examens "de la carcasse témoin sont suspendus à la $96^{\mathrm{e}}$ heure, l'odeur étant insoutenable et la putréfaction trop avancée.

Les résultats obtenus dans le dosage de l'azote basique volatil total semblent montrer une 'évolution régressive entre la $48^{\mathrm{e}}$ heure et la $96^{\text {e }}$ heure pour la carcasse $\mathrm{Pp}$. Ces résultats sont la conséquence ' d'un accroissement très faible du taux; accroissement qui est à la limite inférieure de la précision de la méthode employée (distillation Schloesnig et Aubin). Cet incident ne s'est pas reproduit après l'adoption de la méthode de Conway.
A la 144e heure, les carcasses traitées sont, au point de vue dosage d'azote volatil et numération de germes, au stade de la carcasse témoin à la $72^{\mathrm{e}}$ heure, alors qu'au point de vue aspect et examen organoleptique la différence est beaucoup plus importante. Nous devons signaler que, dès la $72^{e}$ heure, des moisissures se sont développées rapidement sur les carcasses traitées alors qu'elles sont demeurées discrètes sur le témoin. Cette croissance exubérante a pu, en une certaine mesure, contribuer, au delà de la $96^{\mathrm{e}}$ heure, à la protection des carcasses contre la prolifération bactérienne d'origine externe. 


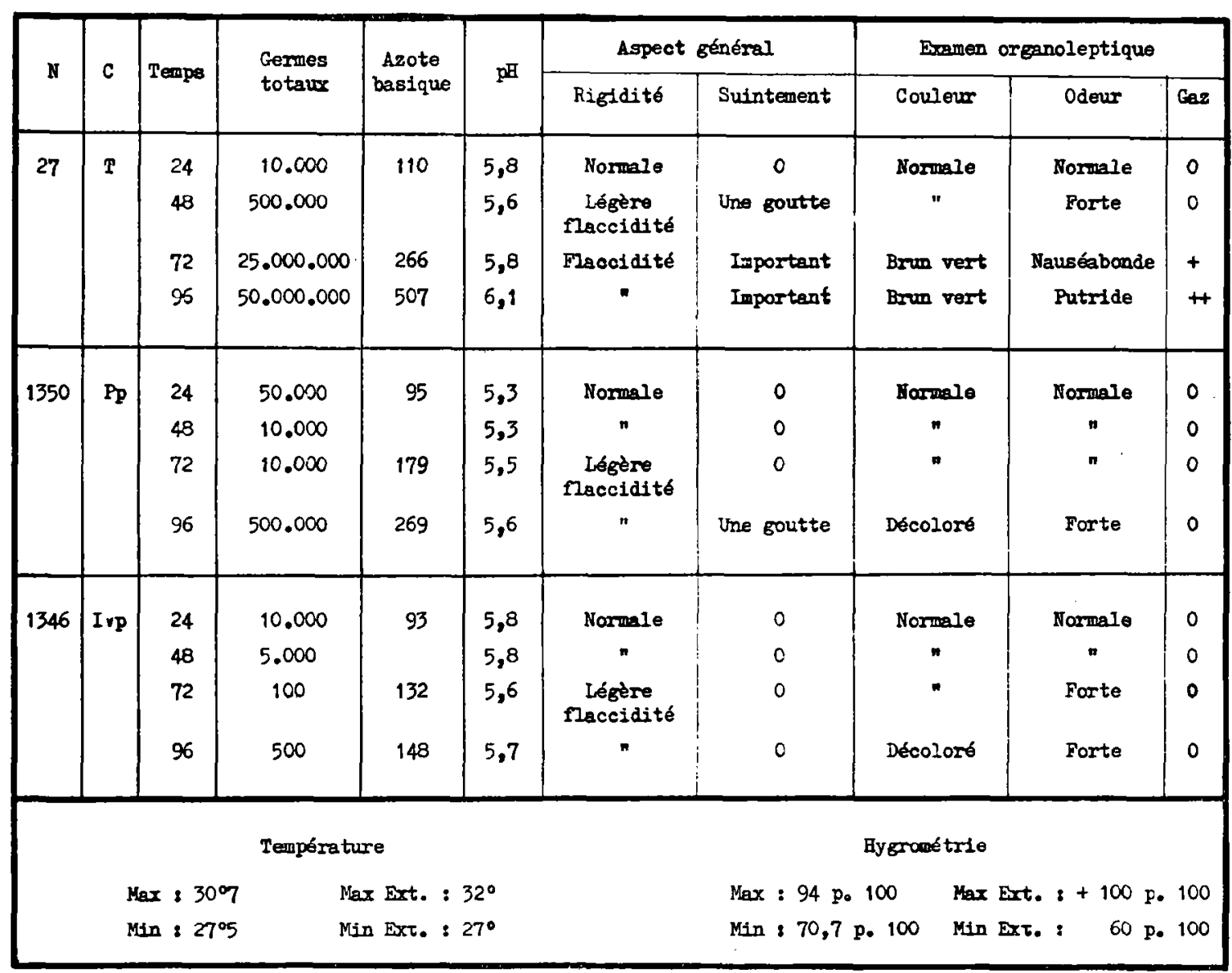

Les dosages d'azote basique volatil total de la $48^{\mathrm{e}}$ heure ne sont pas notés, les flacons contenant les extraits ayant été cassés.

L'ensemble du processus de putréfaction semble avoir été beaucoup plus rapide lors de cette expérimentation. Ce fait peut être en relation avec le climat, la température et le degré hygrométrique étant bien supérieurs à ceux existants au cours des autres expérimentations.

L'efficacité de l'auréomycine semble ici aussi caractéristique.

Les moisissures sont encore plus importantes sur les carcasses traitées que sur la carcasse témoin ; un traitement à l'acide sorbique à raison de 2,5 $\mathrm{g}$ dans $1 / 2$ litre d'eau (solution chauffée à $50^{\circ}$ pour favoriser la solubilité faible) est effectué en même temps que la pulvérisation à l'auréomycine et se montre inefficace contre les moisissures.

Chaque carcasse de l'expérimentation IIl est fendue en deux demi-carcasses : une demicarcasse de chaque animal est maintenue dans les conditions extérieures décrites, l'autre demicarcasse est mise en chambre froide $\mathbf{a}+1^{\circ}$ $+2^{\circ} \mathrm{C}$ jusquau 6-11-1957. A cette date, les trois demi-carcasses sont placées à l'extéricur dans les mêmes conditions que les précédentes et donnent lieu aux mêrmes examens (lot IV). 


\begin{tabular}{|c|c|c|c|c|c|c|c|c|c|}
\hline \multirow{2}{*}{$\mathbf{N}$} & \multirow{2}{*}{ c } & \multirow{2}{*}{ Temps } & \multirow{2}{*}{$\begin{array}{c}\text { Azote } \\
\text { basique }\end{array}$} & \multirow{2}{*}{$\mathrm{pH}$} & \multicolumn{2}{|c|}{ Aspect général } & \multicolumn{3}{|c|}{ Examen organoleptique } \\
\hline & & & & & Rigidité & Suintement & Couleur & Odeur & $\mathrm{Gaz}$ \\
\hline 27 & $\mathbf{T}$ & $\begin{array}{c}0 \\
24 \\
48 \\
72 \\
96 \\
120\end{array}$ & $\begin{array}{c}214 \\
217 \\
392 \\
728 \\
1.029 \\
1.205\end{array}$ & $\begin{array}{l}6,0 \\
5,8 \\
5,9 \\
6,2 \\
6,2 \\
6,4\end{array}$ & $\begin{array}{l}\text { Très ferme } \\
\text { Légère flaccidité } \\
\text { Flaccidité } \\
\text { n } \\
\text { Flaccidité totale } \\
\text { Flacciòité totale }\end{array}$ & $\begin{array}{c}\text { Très sec } \\
0 \\
\text { Quelques gouttes } \\
\text { Important } \\
\text { Important } \\
\text { Très important }\end{array}$ & $\begin{array}{c}\text { Normale } \\
" \\
\text { Verdatre } \\
\text { Brun verdatre } \\
\text { Brun verdatre } \\
"\end{array}$ & $\begin{array}{c}\text { Fade } \\
" \\
\text { Forte } \\
\text { Nauséabonde } \\
\text { Putride } \\
\text { Très putride }\end{array}$ & $\begin{array}{l}0 \\
0 \\
0 \\
+ \\
++ \\
++\end{array}$ \\
\hline 1350 & $P p$ & $\begin{array}{c}0 \\
24 \\
48 \\
72 \\
96 \\
120\end{array}$ & $\begin{array}{l}215 \\
232 \\
298 \\
322 \\
401 \\
493\end{array}$ & $\begin{array}{l}5,7 \\
5,6 \\
5,7 \\
5,8 \\
6,0 \\
6,0\end{array}$ & $\begin{array}{l}\text { Très ferme } \\
\text { Légère flaccidité } \\
\text { Flaccidité } \\
" \\
n\end{array}$ & $\begin{array}{c}\text { Très sec } \\
0 \\
0 \\
0 \\
\text { Une goutte } \\
\text { Quelques gouttes }\end{array}$ & $\begin{array}{c}\text { Normale } \\
n \\
n \\
\text { pale } \\
n \\
n\end{array}$ & $\begin{array}{l}\text { Fade } \\
n \\
\text { Désagréable } \\
\text { Forte } \\
\text { Forte }\end{array}$ & $\begin{array}{l}0 \\
0 \\
0 \\
0\end{array}$ \\
\hline 1346 & $I V p$ & $\begin{array}{c}0 \\
24 \\
48 \\
72 \\
96 \\
120\end{array}$ & $\begin{array}{l}215 \\
224 \\
270 \\
302 \\
392 \\
505\end{array}$ & $\begin{array}{l}6,0 \\
6,0 \\
5,8 \\
5,9 \\
6,1 \\
6,2\end{array}$ & $\begin{array}{l}\text { Très ferme } \\
\text { Légère flaccidité } \\
\text { Légère flaccidité } \\
\text { Flaccidité } \\
n \\
n\end{array}$ & $\begin{array}{c}\text { Très sec } \\
0 \\
0 \\
0 \\
0 \\
\text { Une goutte }\end{array}$ & $\begin{array}{c}\text { Normale } \\
" \\
\text { " } \\
\text { Pâle } \\
\text { Reflet verdâtre }\end{array}$ & $\begin{array}{c}\text { Fade } \\
\text { Désagréable } \\
" \\
\text { Forte }\end{array}$ & $\begin{array}{l}0 \\
0 \\
0 \\
0 \\
0 \\
0\end{array}$ \\
\hline \multicolumn{6}{|c|}{ Tenpérature } & \multicolumn{4}{|c|}{ Hygrométrie } \\
\hline & \multicolumn{3}{|c|}{$\begin{array}{l}\text { Max : } 30^{\circ} \\
\text { Min : } 27^{\circ} 6\end{array}$} & $\begin{array}{l}\text { Max } \\
\text { Min }\end{array}$ & $\begin{array}{l}\text { Ext. : } 31^{\circ} \\
\text { Ext. : } 26^{\circ} 5\end{array}$ & \multicolumn{2}{|c|}{$\begin{array}{l}\text { Max : } 83,2 \text { p. } 100 \\
\text { Min : 60,5 p. } 100\end{array}$} & $\begin{array}{l}\text { t. : } 87 \text { p. } 100 \\
\text { t. : } 57 \text { p. } 100\end{array}$ & \\
\hline
\end{tabular}

Le taux d'azote basique volatil total est déjà élevé à la sortie de la chambre froide. Il est dû vraisemblablement à des phénomènes enzymatiques qui, malgré la température assez basse, ont pu s'effectuer en 23 jours.

A la sortie de la chambre froide, les carcasses présentent un certain degré de dessication superficielle et les moisissures se sont très peu déve- loppées. L'état de conservation apparent est excellent.

Cette expérimentation semble prouver que, même après un séjour assez prolongé des carcasses traitées en chambre froide, leur conservation est meilleure, lorsqu'elles sont replacées dans l'ambiance extérieure, que les témoins, non traités, mais placés par ailleurs dans les mêmes conditions. 
IOT $n^{\circ} \mathrm{V}$ - 25 Novenbre - 30 Novembre 1957

\begin{tabular}{|c|c|c|c|c|c|c|c|c|}
\hline \multirow{2}{*}{ N } & \multirow{2}{*}{$\mathrm{C}$} & \multirow{2}{*}{ Temps } & \multirow{2}{*}{$\begin{array}{c}\text { Azote } \\
\text { basique }\end{array}$} & \multicolumn{2}{|c|}{ Aspect général } & \multicolumn{3}{|c|}{ Ecamen organoleptique } \\
\hline & & & & Rigidité & Suintement & Couleur. & Odeur & $\mathrm{Gaz}$ \\
\hline IF & $\mathrm{T}$ & $\begin{array}{l}24 \\
48 \\
72 \\
96\end{array}$ & $\begin{array}{l}124 \\
227 \\
268 \\
472\end{array}$ & $\begin{array}{l}\text { Normale } \\
\text { Normale } \\
\text { Fleccidité } \\
\text { Flaccidité }\end{array}$ & $\begin{array}{c}0 \\
\text { Une soutte } \\
\text { Inportant } \\
\text { Tres important }\end{array}$ & $\begin{array}{l}\text { Nermale } \\
\text { Normale } \\
\text { Verdâtre } \\
\text { Très verdêtre }\end{array}$ & $\begin{array}{l}\text { Normale } \\
\text { Iégère } \\
\text { Très forte } \\
\text { Fótide }\end{array}$ & $\begin{array}{l}0 \\
0 \\
0 \\
+\end{array}$ \\
\hline IP & p & $\begin{array}{l}24 \\
48 \\
72 \\
96\end{array}$ & $\begin{array}{l}109 \\
199 \\
252 \\
357\end{array}$ & $\begin{array}{l}\text { Normale } \\
\text { Normale } \\
\text { Légère flaccidité } \\
\text { Flaccidité }\end{array}$ & $\begin{array}{c}0 \\
0 \\
\text { Deur gouttes } \\
\text { Quelques gouttes }\end{array}$ & $\begin{array}{c}\text { Normale } \\
\text { Normale } \\
\text { Verdêtre } \\
\text { Verte }\end{array}$ & $\begin{array}{l}\text { Normale } \\
\text { Normale } \\
\text { Forte } \\
\text { Nauséabonde }\end{array}$ & $\begin{array}{l}0 \\
0 \\
0 \\
+\end{array}$ \\
\hline 1396 & $P \mathbf{p}$ & $\begin{array}{r}24 \\
48 \\
72 \\
96 \\
120\end{array}$ & $\begin{array}{l}112 \\
124 \\
134 \\
133 \\
168\end{array}$ & $\begin{array}{l}\text { Normale } \\
\text { Normale } \\
\text { Normale } \\
\text { Juégère flaccidité } \\
\text { Légère flaccidité }\end{array}$ & $\begin{array}{c}0 \\
0 \\
0 \\
\text { One goutte } \\
\text { Dewx gouttes }\end{array}$ & $\begin{array}{l}\text { Normale } \\
\text { Hormale } \\
\text { Hormale } \\
\text { Trace verdátre } \\
\text { Bleutée }\end{array}$ & $\begin{array}{c}\text { Normale } \\
\text { Normale } \\
\text { Légère } \\
\text { Légère } \\
\text { Assez forte }\end{array}$ & $\begin{array}{l}0 \\
0 \\
0 \\
0 \\
0\end{array}$ \\
\hline 1396 & Irp & $\begin{array}{r}24 \\
48 \\
72 \\
96 \\
120\end{array}$ & $\begin{array}{l}120 \\
119 \\
143 \\
161 \\
158\end{array}$ & $\begin{array}{c}\text { Normale } \\
\text { Normale } \\
\text { Normale } \\
\text { Légère flaccidité } \\
\text { Légère flaccidité }\end{array}$ & $\begin{array}{l}0 \\
0 \\
0 \\
0 \\
0\end{array}$ & $\begin{array}{c}\text { Normale } \\
\text { Normale } \\
\text { Normale } \\
\text { Trace bleutée } \\
\text { Trace bleutée }\end{array}$ & $\begin{array}{l}\text { Normale } \\
\text { Normale } \\
\text { Normale } \\
\text { Légère } \\
\text { Légère }\end{array}$ & $\begin{array}{l}0 \\
0 \\
0 \\
0 \\
0\end{array}$ \\
\hline \multicolumn{9}{|c|}{ Tenpérature } \\
\hline & \multicolumn{3}{|c|}{$\begin{array}{l}\text { Max : } 28^{\circ} 6 \\
\text { Min : } 26 \circ 5\end{array}$} & $\begin{array}{l}\text { Max Ext. : } 30^{\circ 5} \\
\text { Min Ext. : } 25^{\circ 5}\end{array}$ & \multicolumn{2}{|c|}{$\begin{array}{l}\text { Max : } 85,8 \text { p. } 100 \\
\text { Min : } 55,3 \text { p. } 100\end{array}$} & $\begin{array}{l}\text { Ext. : } 92 \text { p. } 1 \\
\text { ext. : } 44,5 \text { p. }\end{array}$ & \\
\hline
\end{tabular}

Nous ne constatons aucune différence essentielle entre les carcasses $T$ et $p$, mais une diffé-
rence appréciable entre les carcasses $T$ et $p$ d'une part et $P p$ et Ivp d'autre part. Résultats concordant avec les précédents. 


\begin{tabular}{|c|c|c|c|c|c|c|c|c|}
\hline \multirow{2}{*}{ N } & \multirow{2}{*}{ c } & \multirow{2}{*}{ Tenps } & \multirow{2}{*}{$\begin{array}{c}\text { Azote } \\
\text { basique }\end{array}$} & \multicolumn{2}{|c|}{ Aspect général } & \multicolumn{3}{|c|}{ Examen organoleptique } \\
\hline & & & & Rigidité & Suintement & Couleur & Odeur & Gaz \\
\hline 302 & $\mathrm{~T}$ & $\begin{array}{r}24 \\
48 \\
72 \\
96 \\
120\end{array}$ & $\begin{array}{r}79 \\
126 \\
153 \\
173 \\
\end{array}$ & $\begin{array}{l}\text { Normale } \\
\text { Normale } \\
\text { Normale } \\
\text { Légère flaccidité } \\
\text { Légère flaccidité }\end{array}$ & $\begin{array}{c}0 \\
0 \\
0 \\
\text { Une goutte } \\
\text { Quelques gouttes }\end{array}$ & $\begin{array}{c}\text { Normale } \\
\text { Normale } \\
\text { Normale } \\
\text { Trainée verdâtre } \\
\text { Verte }\end{array}$ & $\begin{array}{l}\text { Normale } \\
\text { Normale } \\
\text { Normale } \\
\text { Forte } \\
\text { Très forte }\end{array}$ & $\begin{array}{l}0 \\
0 \\
0 \\
0 \\
0\end{array}$ \\
\hline 403 & $P p$ & $\begin{array}{r}24 \\
48 \\
72 \\
96 \\
120\end{array}$ & $\begin{array}{r}82 \\
89 \\
99 \\
105\end{array}$ & $\begin{array}{l}\text { Normale } \\
\text { Normale } \\
\text { Normale } \\
\text { Normale } \\
\text { Normale }\end{array}$ & $\begin{array}{l}0 \\
0 \\
0 \\
0 \\
0\end{array}$ & $\begin{array}{l}\text { Normale } \\
\text { Normale } \\
\text { Normale } \\
\text { Normale } \\
\text { Normale }\end{array}$ & $\begin{array}{l}\text { Normale } \\
\text { Normale } \\
\text { Normale } \\
\text { Normele } \\
\text { Normale }\end{array}$ & $\begin{array}{l}0 \\
0 \\
0 \\
0 \\
0\end{array}$ \\
\hline 327 & Pp & $\begin{array}{r}24 \\
48 \\
72 \\
96 \\
120\end{array}$ & $\begin{array}{r}83 \\
94 \\
97 \\
99\end{array}$ & $\begin{array}{l}\text { Normale } \\
\text { Normale } \\
\text { Normale } \\
\text { Normale } \\
\text { Normale }\end{array}$ & $\begin{array}{l}0 \\
0 \\
0 \\
0 \\
0\end{array}$ & $\begin{array}{l}\text { Normale } \\
\text { Normale } \\
\text { Normale } \\
\text { Normale } \\
\text { Normale }\end{array}$ & $\begin{array}{l}\text { Normale } \\
\text { Normale } \\
\text { Normale } \\
\text { Normale } \\
\text { Légère }\end{array}$ & $\begin{array}{l}0 \\
0 \\
0 \\
0 \\
0\end{array}$ \\
\hline & & $\begin{array}{l}\text { ax : } 26 \\
\operatorname{in}: 23\end{array}$ & $\begin{array}{l}\text { Tempe } \\
08\end{array}$ & $\begin{array}{l}\text { ature } \\
\text { Max Ext. : } 28^{\circ} 5 \\
\text { Min Ext.: } 22^{\circ 5}\end{array}$ & $\begin{array}{l}\text { Max : } \\
\text { Min : }\end{array}$ & \begin{tabular}{lll} 
& \multicolumn{2}{c}{ Hygromét } \\
6,2 p. 100 & Max \\
2,8 p. 100 & Min
\end{tabular} & $\begin{array}{l}\text { t. : } 92 \text { p. } 100 \\
\text { t. : } 41 \text { p. } 100\end{array}$ & \\
\hline
\end{tabular}

Les deux carcasses traitées dans cette expérience sont perfusées et pulvérisées.

Le processus général revêt une évolution plus lente, semble-t-il, dans l'ensemble. A la $120^{\circ}$ heure, la carcasse témoin n'est pas encore dans un état de putréfaction avancée. Ceci peut être dû à la température relativement basse et au degré hygrométrique relativement faible, enregistré dans cette expérimentation.

A la $120^{\mathrm{e}}$ heure les carcasses traitées sont en très bon état.

Il y a peu de moisissures sur les carcasses.

Les résultats obtenus sur ce lot sont cependant en concordance avec les résultats des lots précédents.

\section{Interprétation des résultats.}

Nous tirerons certaines déductions de ces divers résultats.

$1^{\circ}$ Le processus de putréfaction des carcasses témoins comme celui des carcasses traitées apparaît lié, quant à leur rapidité d'évolution, aux conditions du milieu ambiant et principalement à la température et à l'hygrométrie. Il ne nous est cependant pas possible de tirer des conclusions définitives, le nombre d'expérimen- tations, d'une part en saison des pluies, d'autre part en saison fraîche, étant insuffisant.

$2^{\circ}$ Les expérimentations $\mathrm{I}$ et $\mathrm{V}$ ne révèlent aucune différence significative entre l'évolution des carcasses témoins et celle des carcasses pulvérisées. Mais ces deux observations sont insuffisantes. Néanmoins, il est intéressant de comparer ces résultats à ceux de Ginsberg et coll. au Kenya (1957). La contradiction apparente qui s'en dégage trouve son explication dans 
l'examen et la comparaison des conditions expérimentales qui ont présidé à ces travaux. Au Kenya, l'expérimentation se termine à la $72^{\mathrm{e}}$ heure et les conditions sont telles que la contamination d'origine externe est primordiale, et tellement importante qu'elle est pratiquement seule en cause. On conçoit ainsi le rôle que peut jouer une pulvérisation en dressant une barrière protectrice superficielle contre cette pollution. Lors de nos expérimentations le problème est inverse. Si la pollution externe n'est pas nulle, elle est demeurée faible et la putréfaction due aux germes internes semble avoir été primordiale. Nos expériences ayant aussi duré plus longtemps ( 96,120 et même 144 heures), la putréfaction interne a eu le temps de se développer et de jouer le rôle principal.

30 Aucune différence essentielle n'est à signaler entre l'évolution des carcasses traitées par perfusion et celles traitées par injection intraveineuse ante mortem. Cette demière méthode, compte tenu de ses avantages techniques, nous semble donc supérieure à la première, d'autant qu’à plusieurs reprises la viande prélevée sur les carcasses perfusées nous est apparue légèrement plus suintante que la viande normale.

$4^{\circ}$ L'efficacité de l'auréomycine semble être certaine dans les conditions d'application décrites, que ce soit en saison des pluies ou en saison fraîche.

$5^{\circ}$ Selon le critère choisi pour évaluer le degré de putréfaction des viandes, l'augmentation du temps de conservation varie sensiblement. Au point de vue organoleptique, le gain de temps est supérieur à 72 heures, bien qu'il soit difficile de comparer l'évolution des carcasses témoins et celles des carcasses traitées. La putréfaction semble en effet évoluer d'une manière différente dans chacun des cas ; pour un même degré d'ètat général, lorsqu'une section est pratiquée dans une masse musculaire, la viande des carcasses traitées semble en bien meilleur état que celle des carcasses témoins. Pour étayer ce jugement, nous examinerons statistiquement, dans le chapitre suivant, les résultats des dosages de l'azote volatil total.

$6^{\circ}$ Les résultats de la numération des germes sont rarement discordants par rapport aux autres et jamais en opposition.

L'étude de la flore nous montre que sur les carcasses traitées, les germes, qui se développent les premiers et en plus grande abondance, sont surtout des bacilles Gram régatif, virant ou non le lactose, des genres Escherichia, Aerobacter, Klebsiella, Alcaligenes, Paracolobactrum, Proteus et très souvent du genre Serratia, mais peu de germes Gram positif et surtout peu ou pas de clostridium, essentiellement putréfiants. En conséquence la putréfaction y est moins explosive, plus lente, moins délabrante surtout parce que sans gaz, que sur le témoin où la présence de certains bacillus et staphylocoques et surtout de clostridies la font évoluer d'une toute autre manière.

$7^{\circ}$ Les tests d'antibiorésistance révèlent un assez grand nombre de souches résistantes à l'auréomycine. Cependant la répartition montre qu'il est sensiblement le même sur les carcasses témoins que sur les carcasses traitées. Toutes les souches résistantes sont des germes Gram négatif, appartenant soit au genre Paracolobactrum, soit au genre Serratia, mais surtout aux genres Proteus et Pseudomonas.

Il ne semble donc pas que le traitement à l'auréomycine soit à l'origine d'une antibiorésistance ; cependant il est évident que ce sont ces souches naturellement résistantes qui apparaissent et se développent le plus rapidement sur les carcasses traitées. Il y a une corrélation certaine entre la non-existence de souches résistantes parmi les germes Gram positif et la bien moindre proportion de ces germes sur les carcasses traitées que sur les carcasses témoins.

$8^{0}$ Aucune interprétation logique ne peut être tirée de la mesure du $\mathrm{pH}$;

$9^{\circ}$ Les résultats du lot IV sont assez intéressants ; ils sembleraient montrer que dans les conditions de l'expérimentation, des carcasses traitées, même après un séjour prolongé en chambre froide ( 23 jours), et encore en bon état, se conservent, une fois remises à la température ambiante, mieux que celles placées dans les mêmes conditions mais non traitées. Cette question devrait être reprise sur un plus grand nombre d'animaux, suivant une variante consistant à congeler les carcasses en tunnel après traitement, et à les conserver en frigorifique à $-20^{\circ}$.

$10^{\circ} \mathrm{Il}$ est à remarquer enfin (lots II et III) que la prolifération de moisissures superficielles est plus intense sur les carcasses traitées que sur les témoins. L'antibiotique en inhibant le développement bactérien en surface semble favoriser l'envahissement par les moisissures (variations du $\mathrm{pH}$ de la viande). 


\section{B. - DOSAGES DE L'AZOTE BASIQUE VOLATIL TOTAL ET ETUDE STATISTIQUE}

AZOTE BASIQUE VOIATIL RÉSULTATS GLOBAUX (Trableau récapitulatif).

TABLEAU VII

\begin{tabular}{|c|c|c|c|c|c|c|c|c|c|c|c|c|}
\hline \multicolumn{3}{|c|}{ Animaux } & \multicolumn{5}{|c|}{ Résultato en $\mathrm{mg} / \mathrm{kg}$ de vignde } & \multicolumn{5}{|c|}{$\begin{array}{l}\text { Résultats en pourcentage, en rame- } \\
\text { nant à } 100 \text { le taux de lo } 24 \mathrm{e} \text { heure }\end{array}$} \\
\hline Catégorie & Lot & Numéro & $\begin{array}{l}\text { A près } \\
24 \mathrm{~h}\end{array}$ & $\begin{array}{l}\text { Après } \\
48 \mathrm{~h}\end{array}$ & $\begin{array}{l}\text { Après } \\
72 \mathrm{~h}\end{array}$ & $\begin{array}{l}\text { Après } \\
96 \mathrm{~h}\end{array}$ & Après & $\begin{aligned} \text { Après } \\
24 \mathrm{~h}\end{aligned}$ & $\begin{array}{r}\text { Après } \\
48 \mathrm{~h}\end{array}$ & $\begin{array}{l}\text { Après } \\
72 \mathrm{~h}\end{array}$ & $\begin{aligned} \text { Après } \\
96 \mathrm{~h}\end{aligned}$ & $\begin{array}{l}\text { Après } \\
120 \mathrm{~h}\end{array}$ \\
\hline Témoins & $\begin{array}{c}I \\
I I \\
I I I \\
V \\
V I\end{array}$ & $\begin{array}{l}1087 \\
209 \\
27 \\
I F \\
302\end{array}$ & $\begin{array}{r}80 \\
110 \\
110 \\
124 \\
79\end{array}$ & $\begin{array}{l}111 \\
123 \\
227 \\
126\end{array}$ & $\begin{array}{l}196 \\
201 \\
226 \\
268 \\
153\end{array}$ & $\begin{array}{l}324 \\
507 \\
472 \\
173\end{array}$ & 380 & $\begin{array}{l}100 \\
100 \\
100 \\
100 \\
100\end{array}$ & $\begin{array}{l}138,8 \\
111,8 \\
181,6 \\
158,9\end{array}$ & $\begin{array}{l}245 \\
182,7 \\
241,8 \\
214,2 \\
199,6\end{array}$ & $\begin{array}{l}294,5 \\
460,9 \\
377,4 \\
218,9\end{array}$ & 450 \\
\hline Pulvérisés & $\begin{array}{l}I \\
V\end{array}$ & $\begin{array}{l}134 \\
\text { IF }\end{array}$ & $\begin{array}{r}80 \\
109\end{array}$ & $\begin{array}{r}56 \\
199\end{array}$ & $\begin{array}{l}186 \\
252\end{array}$ & 357 & 261 & $\begin{array}{l}100 \\
100\end{array}$ & $\begin{array}{c}70 \\
182,4\end{array}$ & $\begin{array}{l}232,5 \\
231,2\end{array}$ & 327,5 & 326,2 \\
\hline $\begin{array}{l}\text { Perfusés et } \\
\text { pulvérisés }\end{array}$ & $\begin{array}{c}\text { II } \\
\text { III } \\
\text { V } \\
\text { VI } \\
\text { VII }\end{array}$ & $\begin{array}{r}1083 \\
1350 \\
1393 \\
405 \\
327\end{array}$ & $\begin{array}{r}122 \\
95 \\
112 \\
82 \\
85\end{array}$ & $\begin{array}{r}124 \\
89 \\
94\end{array}$ & $\begin{array}{r}145 \\
179 \\
134 \\
99 \\
97\end{array}$ & $\begin{array}{r}140 \\
269 \\
133 \\
105 \\
99\end{array}$ & 150 & $\begin{array}{l}100 \\
100 \\
100 \\
100 \\
100\end{array}$ & $\begin{array}{l}118,8 \\
110,7 \\
108,5 \\
110,3\end{array}$ & $\begin{array}{l}118,8 \\
188,4 \\
120 \\
121,4 \\
133,8\end{array}$ & $\begin{array}{l}114,7 \\
283,1 \\
118,8 \\
127,8 \\
116,8\end{array}$ & 122,9 \\
\hline $\begin{array}{l}\text { Pulvérisés et } \\
\text { injection } \\
\text { intra-veineuse }\end{array}$ & $\begin{array}{l}\text { II } \\
\text { III } \\
\mathrm{V}\end{array}$ & $\begin{array}{r}132 \\
1346 \\
1396\end{array}$ & $\begin{array}{r}122 \\
93 \\
120\end{array}$ & 140 & $\begin{array}{l}145 \\
132 \\
143\end{array}$ & $\begin{array}{l}145 \\
148 \\
161\end{array}$ & 166 & $\begin{array}{l}100 \\
100 \\
100\end{array}$ & 98,8 & $\begin{array}{l}118,8 \\
141,9 \\
119\end{array}$ & $\begin{array}{l}118,8 \\
159,1 \\
134,1\end{array}$ & 131,6 \\
\hline
\end{tabular}

Dans ce tableau nous avons regroupé les résultats des dosages de l'azote basiquc volatil total en les classant par catégorie : témoins, pulvérisés, perfusés-pulvérisés, intraveineux-pulvérisés, sans tenir compte des lots.

La dispersion des résultats pour un même temps, dans chaque catégorie, est parfois importante du fait de la non-homogénéité des animaux et de la variabilité des conditions extérieures; aussi elle peut nuire à l'interprétation des résultats. Pour limiter cette dispersion nous avons ramené arbitrairement dans la partie droite du tableau le taux d'azote basique volatil, à 100 , pour toutes les carcasses, au temps 24 heures. Nous n'étudierons plus la valeur absolue des taux d'azote volatil, mais l'accroissement de ce taux en pourcentage par rapport au taux à la 24 e heure.

Les calculs statistiques suivants porteront sur les valeurs ainsi ajustées.

Les résultats au delà de la $96^{\mathrm{e}}$ heure sont trop peu nombreux pour faire l'objet d'une étude statistique, cette dernière ne sera donc pas faite après la $96^{\mathrm{e}}$ heure.: 
SZOTE BASIQUE VOLATIL

TABLEAU VIII

RÉSULTATS DES MOYENNES DE CHAQUE CATÉGORIE

\begin{tabular}{|c|c|c|c|c|}
\hline $\begin{array}{c}\text { Temps } \\
\text { en heures }\end{array}$ & Témoins & Pulvérisé & $\begin{array}{c}\text { Pulvérisé } \\
\text { et perfusé }\end{array}$ & $\begin{array}{c}\text { Pulvérisé } \\
\text { et 1.v. }\end{array}$ \\
\hline 24 & 100 & 100 & 100 & 100 \\
48 & 170,44 & 126,20 & 112,07 & 106,75 \\
72 & 216,66 & 231,85 & 132,48 & 126,57 \\
96 & 337,92 & 327,50 & 152,24 & 137,33 \\
\hline
\end{tabular}

REPRÉSENTATION GRAPHIQUE DES RESULTATS : (graphique 1)

La teneur en azote basique volatil triple entre 24 et 96 heures pour le lot témoin et le lot pulvérisé. Il semble apparaître un retard à la produc- tion d'azote basique volatil de 24 à 48 heures pour le lot pulvérisé.

La production d'azote basique volatil est moitié moindre pour les lots pulvérisé-perfusé et pulvérisé-intraveineux que pour les lots témoins et pulvérisés.

En raison du nombre réduit des carcasses examirées, de la faible production d'azote dans les deux derniers lots, les résultats sont analysés statistiquement :

$1^{\mathrm{o}}$ pour voir si la production d'azote est bien une fonction du temps ;

$2^{\circ}$ si cette production peut être représentée par une fonction mathématique simple;

$3^{0}$ si les différentes représentations mathématiques traduisert l'influerce des différents traitements appliqués.

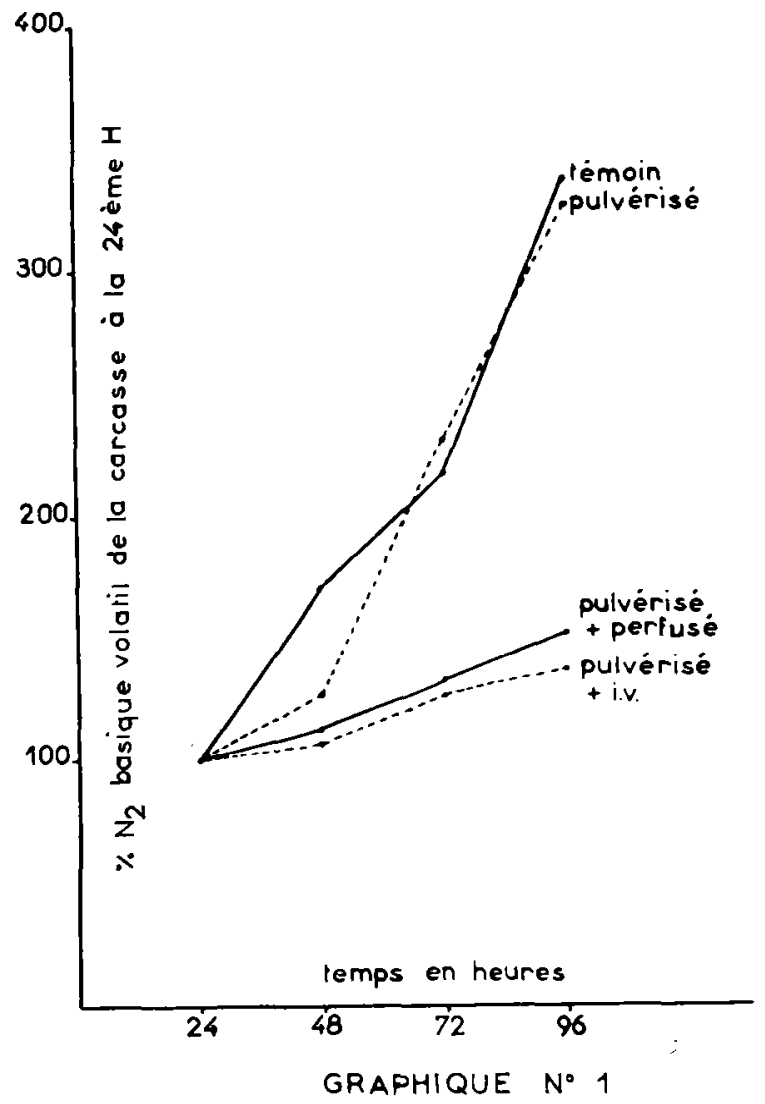




\begin{tabular}{|c|c|c|c|c|c|c|}
\hline Lotg & $\begin{array}{l}\text { Somme } \\
\text { des } \\
\text { carrés }\end{array}$ & $\begin{array}{l}\text { Degrés } \\
\text { de } \\
\text { liberté }\end{array}$ & Variance & $\frac{s^{2} c}{s^{2} e}$ & Souil & $\begin{array}{l}\text { Action } \\
\text { du } \\
\text { temps }\end{array}$ \\
\hline $\begin{array}{l}\text { Lot témoin } \\
\text { Variation totale } \\
\text { Variation entre classes de temps } \\
\text { Variation résiduelle }\end{array}$ & $\begin{array}{r}189749,828 \\
141213,518 \\
48536,310\end{array}$ & $\begin{array}{r}19 \\
3 \\
16\end{array}$ & $\begin{array}{l}s^{2} c=47071,173 \\
s^{2} e=3033,519\end{array}$ & 15,517 & 3,24 & Significative \\
\hline $\begin{array}{l}\text { Lot pulvérisé } \\
\text { Variation totale } \\
\text { Variation entre classes de temps } \\
\text { Variation résiduelle }\end{array}$ & $\begin{array}{rr}52 & 001,630 \\
45 & 683,905 \\
6 & 317,725\end{array}$ & $\begin{array}{r}10 \\
3 \\
7\end{array}$ & $\begin{array}{l}s^{2} c=15227,968 \\
s^{2} e=902,532\end{array}$ & 16,872 & 4,35 & Significative \\
\hline $\begin{array}{l}\text { Lot pulvéxisé-perfusé } \\
\text { Variation totale } \\
\text { Variation entre classes de temps } \\
\text { Variation résiduelle }\end{array}$ & $\begin{array}{rr}33 & 291,646 \\
7 & 779,577 \\
25 & 512,069\end{array}$ & $\begin{array}{r}18 \\
3 \\
15\end{array}$ & $\begin{array}{l}S^{2} c=2593,192 \\
S^{2}=1700,805\end{array}$ & 1,524 & 3,29 & $\begin{array}{l}\text { Non } \\
\text { significative }\end{array}$ \\
\hline $\begin{array}{l}\text { Lot pulvérisé + Intra-veineuse } \\
\text { Variation totale } \\
\text { Variation entre classes de temps } \\
\text { Variation résiduelle }\end{array}$ & $\begin{array}{l}3870,148 \\
2563,222 \\
+306,926\end{array}$ & $\begin{array}{r}10 \\
3 \\
7\end{array}$ & $\begin{array}{l}S^{2} c=854,407 \\
s^{2} e=186,704\end{array}$ & 4,576 & 4,35 & Signiflcative \\
\hline
\end{tabular}

Sauf pour le lot pulvérisé-perfusé, la variance liée au temps est significativement plus élevée que la variance de l'erreur (table de Snedecor au point 5 p. 100). Le temps intervient significativement dans la production de l'azote basique volatil.

Pour le lot pulvérisé-perfusé, deux cas se présentent : ou bien l'auréomycine entrave la production d'azote, ce qui n'est pas certain puisque les valeurs moyennes traduisent une augmentation de la teneur en azote, ou bien les résultats obtenus sont trop dispersés et l'augmentation observée entre dans les limites des erreurs d'échantillonnage. Il fera l'objet d'une étude particulière. 


\section{Essais de représentation mathématique de l'évolution du phénomène "régression linéaire "}

10) LOT TÉMOIN : Coefficient de régression : $b_{2} 3,099$

Analyse de la variance en vue du test de

linéarité de la représentation mathématique

\begin{tabular}{|c|c|c|c|c|c|c|}
\hline Origine de la variation & $\begin{array}{l}\text { Somme des } \\
\text { carrés }\end{array}$ & $\begin{array}{l}\text { Degrés de } \\
\text { liberté }\end{array}$ & Variances & $s^{2} D / s^{2} e$ & Seuil & $\begin{array}{l}\text { Test de } \\
\text { linéarité }\end{array}$ \\
\hline $\begin{array}{l}\text { Régression linéaire } \\
\text { Déviatione par rapport } \\
\text { à la droite }\end{array}$ & $\begin{array}{r}135808,243 \\
5405,275\end{array}$ & 1 & $s^{2}=2702,630$ & \multirow{3}{*}{0,891} & \multirow{3}{*}{3,63} & \multirow{3}{*}{ Positif } \\
\hline $\begin{array}{c}\text { Entre classes de temps } \\
\text { Fésiduelle }\end{array}$ & $\begin{array}{r}14 h \quad 213,518 \\
48536,310\end{array}$ & $\begin{array}{r}3 \\
16\end{array}$ & $s^{2} e=3033,519$ & & & \\
\hline Totale & 189749,828 & 19 & & & & \\
\hline
\end{tabular}

Variance du coefficient de régression : $\mathrm{S}^{2} \mathrm{~b}=$ 0,2119 .

Ecart type du coefficient de régression : $\mathrm{S} \mathrm{b}=$ $\pm 0,4604$.

Variance du coefficient de position : $\mathrm{S}^{2} \mathbf{a}=$ 149,84 .

Ecart type du coefficient de position : $\mathrm{S}$ a $=$ $\pm 12,24$.

Forme définitive de la régression linéaire : $\mathrm{Y}^{\circ} \%=19,58( \pm 12,24)+3,099( \pm 0,460) \mathrm{X}$

Entre 24 et 96 heures l'azote basique volatil augmente par heure de 3,099 ( $\pm 0,460)$ p. 100 de la teneur de la carcasse au temps 24 heures (graphique 2).

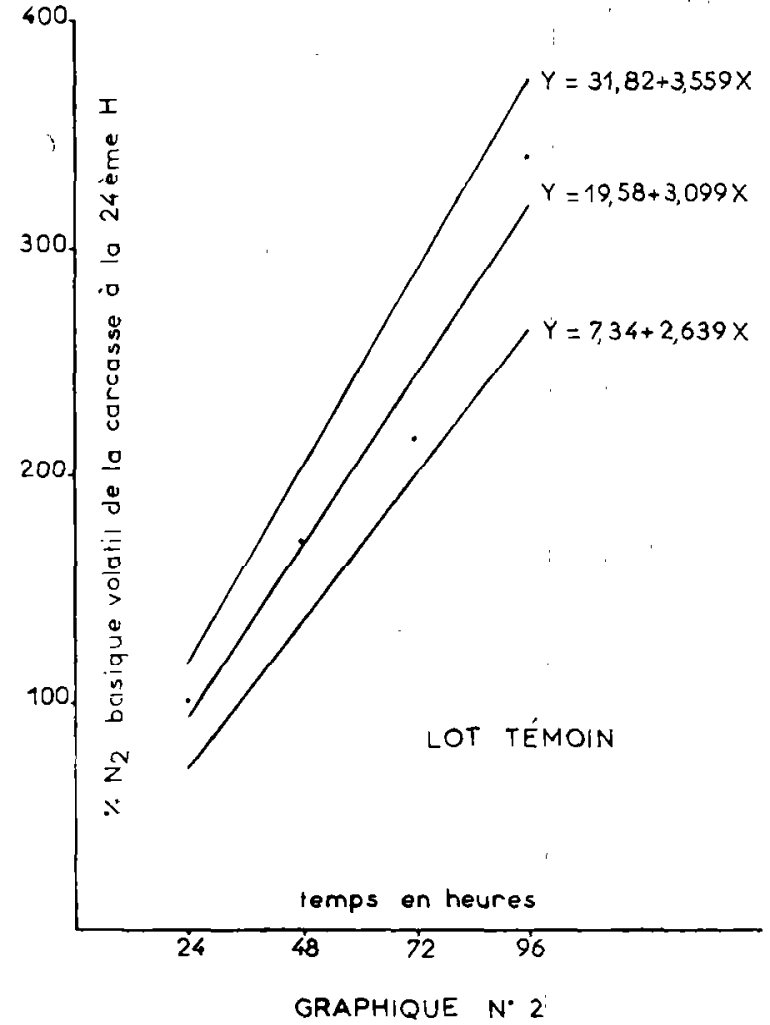


20) LOT PULVERISÉ : Coefficient de régression : $v=3,160$

Analyse de la variance en vue du test de

linéarité de la représentation mathématique

\begin{tabular}{|c|c|c|c|c|c|c|}
\hline Origine de la variation & $\begin{array}{l}\text { Some des } \\
\text { carrés }\end{array}$ & $\begin{array}{l}\text { Degrés de } \\
\text { liberté }\end{array}$ & Variances & $S^{2} D / s^{2} e$ & Sewil & $\begin{array}{l}\text { Test de } \\
\text { linéarité }\end{array}$ \\
\hline $\begin{array}{l}\text { Régression linéaire } \\
\text { Déviations par rapport } \\
\text { a la droite }\end{array}$ & $\begin{array}{l}42729,794 \\
2954,111\end{array}$ & 1 & $s^{2} D=1477,055$ & \multirow{3}{*}{1,636} & \multirow{3}{*}{4,74} & \multirow{3}{*}{ Positif } \\
\hline $\begin{array}{c}\text { Entre classes de temps } \\
\text { Résiduelle }\end{array}$ & $\begin{array}{r}45683,905 \\
6317,725\end{array}$ & $\begin{array}{l}3 \\
7\end{array}$ & $s^{2} e=902,532$ & & & \\
\hline Totale & 52001,630 & 10 & & & & \\
\hline
\end{tabular}

Variance du coefficient de régression : 0,433: Ecart type du coefficient de régression : $\pm 0,658$.

Variance du coefficient de position : 264,91

Ecart type du coefficient de position : $\pm 16,27$. $\mathrm{Y} \%=4,309( \pm 16,27)+3,160( \pm 0,433) \mathrm{X}$

Entre la $24^{\mathrm{e}}$ et la $96^{\mathrm{e}}$ heure, l'azote basique volatil augmente par heure de 3,160 ( $\pm 0,432$ ) p. 100 de la teneur de la carcasse au temps 24 heures (graphique 3).

Forme définitive de la régression linéaire :

30) LOT PULVERISÉ + INTRAVEINEUX : Soefficient de régression : $b=0,542$

Analyse de la variance et test de linéarité

TABLEAU XII

\begin{tabular}{|c|c|c|c|c|c|c|}
\hline Origine de la variation & $\begin{array}{c}\text { Sonme des } \\
\text { carrés }\end{array}$ & $\begin{array}{l}\text { Degrés de } \\
\text { liberté }\end{array}$ & Variances & $s^{2} D / s^{2} e$ & Seuil & $\begin{array}{l}\text { Test de } \\
\text { linéarité }\end{array}$ \\
\hline $\begin{array}{c}\text { Régression linéaire } \\
\text { Déviation par rapport à } \\
\text { la droite }\end{array}$ & $\begin{array}{r}2493,940 \\
69,282\end{array}$ & 1 & $S^{2} D=34,641$ & \multirow{2}{*}{0,185} & \multirow{2}{*}{4,74} & \multirow{2}{*}{ Positif } \\
\hline $\begin{array}{c}\text { Entre classes de temps } \\
\text { Résiduelle }\end{array}$ & $\begin{array}{l}2563,222 \\
1306,926\end{array}$ & $\begin{array}{l}3 \\
7\end{array}$ & $S^{2} e=186,704$ & & & \\
\hline
\end{tabular}

Variance du coefficient de régression : 0,018. Forme définitive de la régression linéaire : Ecart type de coefficient de régression : $Y=85,543( \pm 3,728)+0,542( \pm 0,134) \mathrm{X}$. $\pm 0,1342$.

Variance du coefficient de position : 13,901.

Entre la $24^{\mathrm{e}}$ heure ct la $96^{\mathrm{e}}$ hcurc, le taux

Ecart type du coefficient de position : $\pm 3,728$. d'azote basique volatil augmente de 0,542 $( \pm 0,134)$ p. 100 de la teneur de la carcasse au temps 24 heures (graphique 4). 


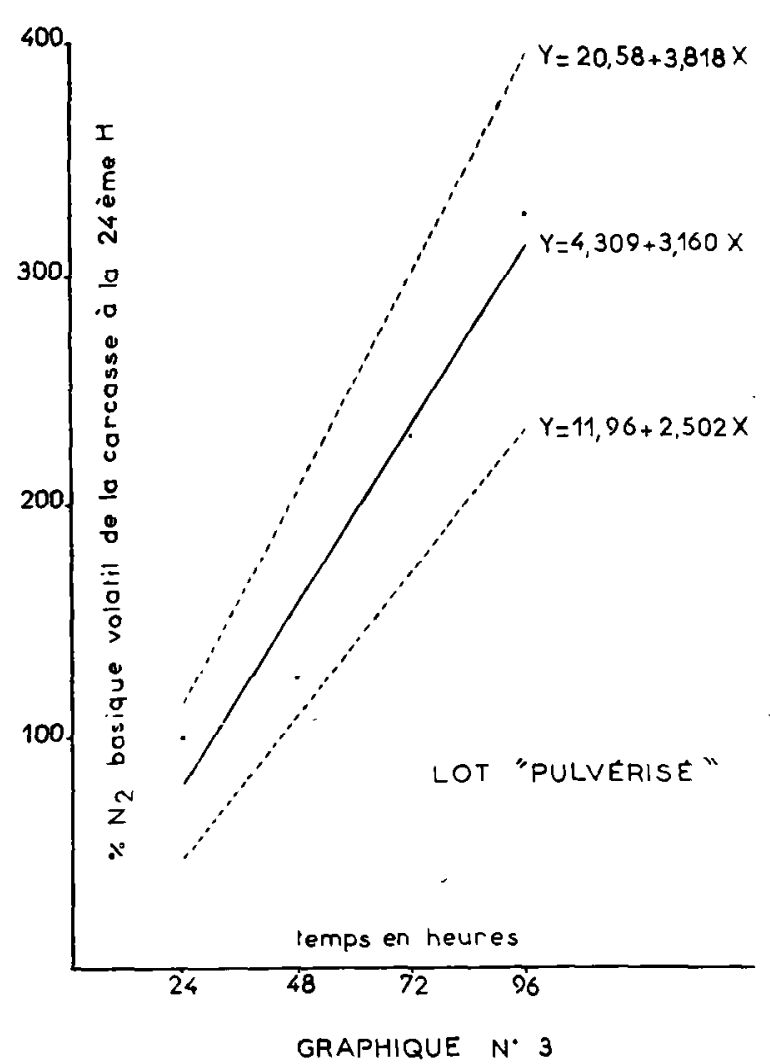

\section{Efficacité comparée des différents traitements}

Elle peut être déduite de l'examen des coeffcients de régression et de la signification de leurs écarts :

Les enrichissements de la carcasse en azote basique volatil ne sont pas significativement différents entre le lot témoin et le lot pulvérisé.

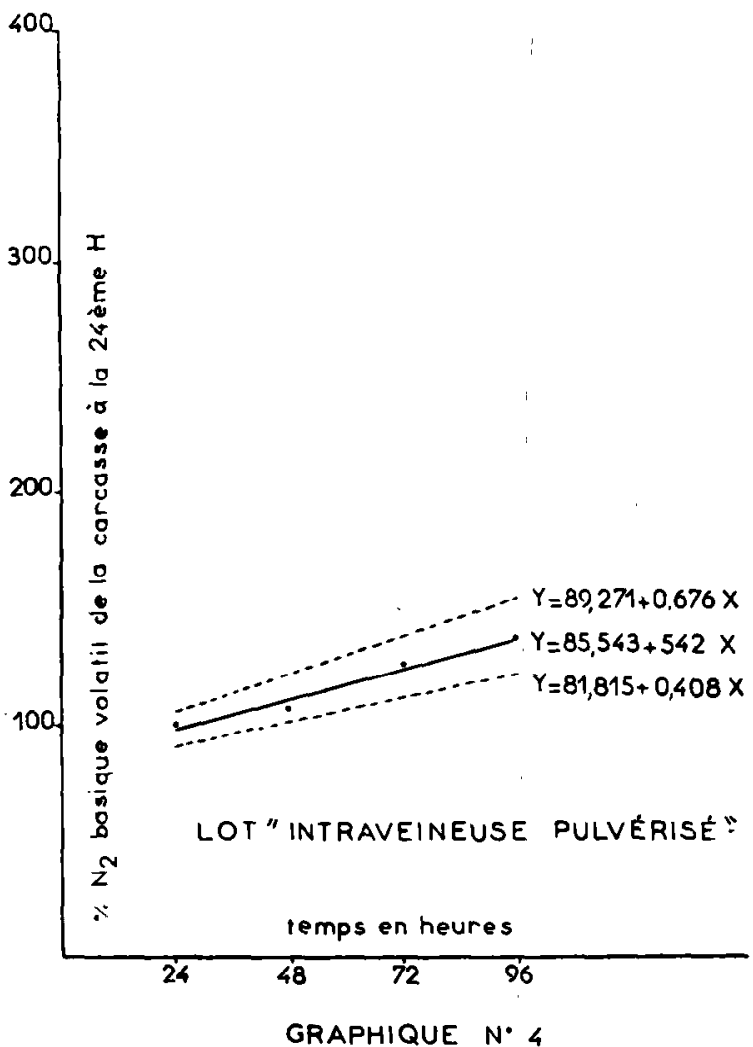

La pulvérisation d'auréomycine paraît inefficace. $\mathrm{Ce}$ résultat demande cependant confirmation en raison de la dispersion des résultats obtenus avec les carcasses pulvérisées.

L'administration intraveineuse de l'antibiotique provoque un ralentissement très net de la production d'azote basique volatil qui devient de 4 à 9 fois plus faible.

TABLEAU XIII

\begin{tabular}{|c|c|c|c|}
\hline & TÉrOIN & PUlvÉRISÉ & INTRA-VEINDIX \\
\cline { 2 - 4 } Coefficient de pente & 3,099 & 3,160 & 0,542 \\
Variance du coefficient de pente & 0,212 & 0,433 & 0,010 \\
Différence des coefficients de pente & - & 0,061 & 2,557 \\
variance de la différence & - & 0,645 & 0,230 \\
Ecart type de la différence & - & 0,0031 & 0,4796 \\
Différence des pentes & - & 0,07 & 5,33 \\
Ecart type de la différence & - & Non significative & Significative \\
Signification des éoarts & - & & \\
\hline
\end{tabular}




\section{Cas du lot perfusé-pulvérisé}

Nous avons vu qu'il n'y avait pas dans ce cas d'enrichissement significatif en azote volatil basique en raison de la dispersion des résultats obtenus.

L'examen de ces résultats montre que cette dispersion est due en grande partie aux résultats obtenus au cours de la saison des pluies.

L'analyse statistique est reprise en ne tenant compte que des essais effectués en saison sèche et en les comparant aux témoins placés dans les mêmes conditions.

ACTION DU TEMPS DANS LA PRODUCTION D'AZOTE BASIQUE VOLATIL

TABLEAD XV

\begin{tabular}{|c|c|c|c|c|c|c|}
\hline . & $\begin{array}{l}\text { Somme des } \\
\text { carrés }\end{array}$ & $\begin{array}{l}\text { Degrés de } \\
\text { liberté }\end{array}$ & Variances & $s^{2} c / s^{2} e$ & Seuil & Action du temps \\
\hline $\begin{array}{c}\text { TFMOIN } \\
\text { Variation totale } \\
\text { Variation entre classes } \\
\text { Variations résiduelles }\end{array}$ & $\begin{array}{r}104715,758 \\
77126,355 \\
27589,403\end{array}$ & $\begin{array}{r}- \\
16 \\
3 \\
13\end{array}$ & $\begin{array}{l}S^{2} c=25708,785 \\
s^{2} e=2122,262\end{array}$ & 12,113 & 3,41 & Significative \\
\hline $\begin{array}{l}\text { PERrUSE } \\
\text { Variation totale } \\
\text { Variation entre classes } \\
\text { Variations résiduelles }\end{array}$ & $\begin{array}{l}162,704 \\
952,915 \\
209,789\end{array}$ & $\begin{array}{c}15 \\
3 \\
12\end{array}$ & $\begin{array}{l}s^{2} c=317,638 \\
s^{2} \theta=17,482\end{array}$ & 18,169 & 3,49 & Significative \\
\hline
\end{tabular}

Étude des régressions linéaires des lots " témoin " et "perfusé " (saison sèche).

10) TEMOIN : Coefficient de régression : $\mathrm{b}=2,594$

Analyse de la variance et test de linéarité :

TABLEAO XVI

\begin{tabular}{|c|c|c|c|c|c|c|}
\hline Origine de la variation & $\begin{array}{l}\text { Somrne des } \\
\text { carrés }\end{array}$ & $\begin{array}{l}\text { Degrés de } \\
\text { liberté }\end{array}$ & Variances & $s^{2} D / s^{2} e$ & Seuil & $\begin{array}{l}\text { Test de } \\
\text { linéarité }\end{array}$ \\
\hline $\begin{array}{c}\text { Régression linéaire } \\
\text { Déviation par rapport à } \\
\text { la droite }\end{array}$ & $\begin{array}{l}75691,930 \\
1434,425\end{array}$ & 1 & $S^{2} D=717,212$ & \multirow{3}{*}{0,338} & \multirow{3}{*}{3,81} & \multirow{3}{*}{ Positif } \\
\hline $\begin{array}{c}\text { Entre classes de temps } \\
\text { Résiduelle }\end{array}$ & $\begin{array}{l}77126,355 \\
27589,403\end{array}$ & $\begin{array}{c}3 \\
15\end{array}$ & $s^{2} e=2122,262$ & & & \\
\hline Totale & 104715,758 & 16 & & & & \\
\hline
\end{tabular}


Variance du coefficient de régression : 0,172 .

Ecart type du coefficient de régression : $\pm 04,147$.

Variance du coefficient de position : 113,818.

Ecart type du coefficient de position : 10,67.
Forme définitive de la régression :

$Y=38,618( \pm 10,67)+2,594( \pm 0,414) X$

Entre la $24^{\mathrm{e}}$ et la $96^{\mathrm{e}}$ heure, le taux de l'azote basique volatil augmente par heure de 2,594 $( \pm 0,414)$ p. 100 de la teneur de la carcasse à la $24^{\mathrm{e}}$ heure (graphique 5) .

20) IOT PERFUSÉ (Saison sèche) : Coefficient de régression : $b=0,270$

Analyse de la variance et test de linéarité .

TABLEAU XVII

\begin{tabular}{|c|c|c|c|c|c|c|}
\hline Origine de la variation & $\begin{array}{l}\text { Somme des } \\
\text { carrés }\end{array}$ & $\begin{array}{l}\text { Degrés de } \\
\text { liberté }\end{array}$ & Variances & $S^{2} D / s^{2} e$ & Seuil & $\begin{array}{l}\text { Test de } \\
\text { linéarité }\end{array}$ \\
\hline $\begin{array}{l}\text { Régression linéaire } \\
\text { Dóriation par rapport à } \\
\text { la droite }\end{array}$ & $\begin{array}{l}834,048 \\
118,867\end{array}$ & 1 & $S^{2} D=59,433$ & \multirow{3}{*}{3,399} & \multirow{3}{*}{3,89} & \multirow{3}{*}{ Positif } \\
\hline $\begin{array}{l}\text { Entre classes } \\
\text { Résiduelle }\end{array}$ & $\begin{array}{l}952,915 \\
209,789\end{array}$ & $\begin{array}{r}3 \\
12\end{array}$ & $S^{2} e=17,482$ & & & \\
\hline Totale & 1162,704 & 15 & & & & \\
\hline
\end{tabular}

Variance du coefficient de régression : 0,002. Forme défnitive de la régression :

Ecart type du coefficient de régression : $Y=96,325( \pm 1,211)+0,270( \pm 0,044) X$. $\pm 0,044$.

Variance du coefficient de position : 1,467. Ecart type du coefficient de position : $\pm 1,211.24$ heures (graphique 6).

De la $24^{\mathrm{e}}$ à la $96^{\mathrm{e}}$ heure, le taux d'azote basique volatil augmente par heure de $0,270( \pm 0,044)$ p. 100 de la teneur de la carcasse au temps

\section{Efficacité de la perfusion}

Examen des coefficients de régression et signification de leurs écarts. (Tableau XVIII).

La perfusion provoque un ralentissement significatif de la production d'azote basique volatil qui devient de 7 à 13 fois plus faible sur la carcasse traitée.
TABLEAU XVIII

\begin{tabular}{|c|c|c|}
\hline & TËOIN & PERFUEE \\
Coefficient de régression & 2,594 & 0,270 \\
\cline { 2 - 3 } Variance du coefficient & & \\
de Iégression & 0,172 & 0,002 \\
Différence des coefficients & & 2,324 \\
de régression & - & 0,174 \\
Variance de cette différence & - & 0,417 \\
Différence des pentes & - & 5,573 \\
Ecart type de la différence & & Boart \\
Signification des écarts & & significatif \\
\hline
\end{tabular}



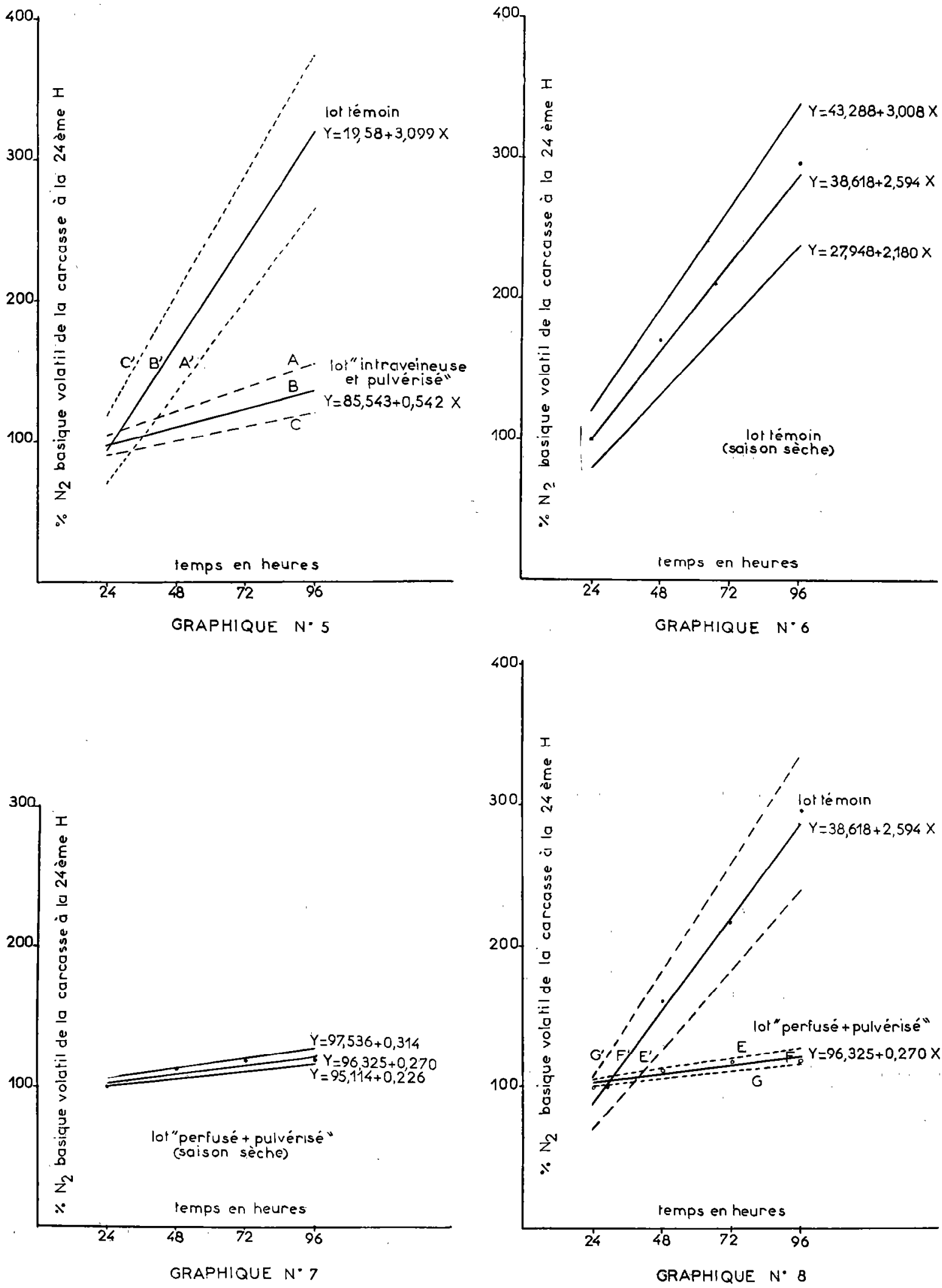


\section{Interprétation des résultats.}

Nous ne ferons que peu de commentaires sur ces résultats. Dans leur rigueur mathématique, ils prouvent l'efficacité de l'antibiotique employé, bien que l'épreuve choisie, parce qu'elle semble transcrire fidèlement l'état exact de décomposition de la viande, apparaisse comme la plus sévère.

Cependant ces résultats peuvent être exprimés plus objectivement par le retard à la putréfaction (exprimé en heures) que le traitement permet d'obtenir à la $96^{\mathrm{e}}$ heure.

Pour cela, il suffit de faire la différence des abcisses, c'est-à-dire des temps, obtenues en donnant aux taux de l'azote basique, dans les équations $\mathrm{du}$ lot traité considéré d'une part, et du lot témoin correspondant d'autre part, la valeur du taux d'azote du lot traité à la 96e heure.

Le retard moyen est donné en calculant cette différence sur les équations des valeurs moyennes d'azote volatil des deux lots, le retard maximum en le calculant sur l'équation des valeurs maxima possibles du lot traité et sur l'équation des valeurs minima possibles du lot témoin, enfin le retard minimum en faisant cette même opération sur l'équation des valeurs minima possibles du lot traité et sur celle des valeurs maxima possibles du lot témoin.

Une représentation graphique de ce calcul est donné dans le graphique 5 pour le lot " intraveineux +-pulvérisé " par les points $A-A^{\prime}-$ B-B' - C-C', et dans le graphique 8 pour le lot " perfusé + pulvérisé " par les points $\mathrm{D}-\mathrm{D}$ ' E-E' - F-F'.

Pour le premier le retard minimum est de 40 heures, maximum de 71 heures, moyen de 48 heures. Pour le second le retard minimum est de 48 heures, maximum de 69 heures et moyen de 59 heures.

Les représentations graphiques du lot intraveineux-pulvérisé et du lot perfusé-pulvérisé ne peuvent pas être comparées exactement étant donné que les résultats de la saison des pluies ne sont pas retenus dans le second.

Il n'en demeure pas moins que les résultats obtenus sont très voisins les uns des autres et comparables aux résultats précédents bien qu'ayant toute la rigueur requise.

\section{DISCUSSION}

Il est certain que les mesures habituelles préconisées par les hygiénistes pour une bonne conservation des viandes gardent et garderont toute leur valeur. Ces expériences montrent cependant que leur effet est limité dans le temps, surtout en Afrique où leur application stricte demeure difficile, le mode d'abattage local entraînant par exemple une bactériémie certainement importante. Ainsi, la pollution profonde est loin d'être négligeable : l'efficacité incontestable de l'antibiotique employé par voie interne et son inefficacité par voie externe lorsque la pollution superficielle est réduite au minimum le prouve. Ginsbertg et coll. (1957) affirment que les germes pénètrent dans les couches profondes à la faveur des espaces conjonctifs, des vaisseaux lymphatiques, des vaisseaux sanguins, etc. Ceci n'est entièrement vrai que lorsque les carcasses subissent des manipulations non hygiériques et sont transportées dans de mauvaises conditions, comme ce fut le cas dans leurs expériences. La protection superficielle devient alors primordiale.

De son côté Sacchi en 1955, cité par Ginsberg, aurait obtenu à Cuba de bons résultats en utilisant la voie péritonéale, mais ses conditions d'expérimentation sont encore particulières. Il est donc difficile de comparer les résultats obtenus par les différents auteurs, et les modes d'utilisation efficaces de l'auréomycine ou de tout autre antibiotique actif sont directement tributaires des conditions d'expérimentation. Il serait vain de vouloir porter un jugement définitif dans l'ignorance de ces dernières. Par suite ce sont les conditions d'exploitation de la viande qui dictent la conduite à suivre et la méthode à employer.

Parmi les méthodes utilisant la voie interne, nous ne retiendrons pas la perfusion ; nous avons vu ce qu'il fallait en penser. L'injection intraveineuse, très voisine de l'intrapéritonéale employée par certains auteurs, présente cependant l'avantage sur cette dernière d'entraîner moins de perte d'antibiotique et une meilleure répartition générale. Nous ne sommes pas de l'avis de Ginsberg et coll. qui voient dans son application de grosses difficultés de contention et d'organisation. Bien que l'opération soit plus délicate qu'une simple injection sous-cutanée, et plus lente, un seul opérateur avec quelques aides pour la contention, travaillant dans un Kraal avec couloir, peut traiter en peu de temps plus de bovins que pourrait en absorber un abattoir d'Afrique.

Il va sans dire que nous ne rejetons pas pour autant la méthode par pulvérisation car elle permet une lutte efficace contre la pollution superficielle et par la suite elle peut être un adjuvant appréciable à la méthode précédente 
lorsque les contaminations d'origine externe deviennent massives. Elle peut d'ailleurs s'intercaler très facilement dans les manipulations d'abaltage sans en retarder le cours.

L'antibiotique ne peut cependant en aucun cas remplacer le froid pour la conservation de la viande en carcasses. Il ne peut être qu'un complément qui en prolongera l'emploi ou suppléera à son absencc pendent un temps ccpcrident toujours assez court. Si certains auteurs ont vu, à juste titre, dans l'emploi de l'auréomycine un moyen de conservation de la qualité de la viande au niveau de sa commercialisation, d'autres buts d'utilisation peuvent être envisagés. Au cours de l'expérience du lot IV, les carcasses treitées semblent s'être mieux comportées une fois replacées dans le milicu ambiant que la carcasse témoin et ceci malgré 23 jours de chambre froide à $2^{\circ} \mathrm{C}$. L'antibiotique pourrait, si ce fait est confirmé, être utilisé sur les carcasses congelées ou non, qui auraient à subir des ruptures de la chaîne du froid, comme il est trop fréquent de le constater en de nombreux points de transit africains, ports, gares, ou aérodromes.

Quant aux risques engendrés par l'absorption possible de doses importantes d'auréomycine lors de la consommation de viande récemment traitée et peu cuite, la majorité des auteurs les considèrent comme négligeables. Il faudrait consommer des quantités très importantes de viande non cuite pour absorber une dose thérapeutique d'antibiotique, sinon une dose active. Cependant, la question des antibiotiques devrait être examinéz dans son ensemble. Leurs utilisations dans des buts non thérapeutiques se multiplient et les causes d'absorption en augmentant (Velu 1957) posent, à juste titre, quelques inquiétudes aux hygiénistes. L'emploi des antibiotiques dans un but non thérapeutique devrait être codifié pour éviter leur usage inconsidéré qui pourrait devenir dangereux entre les mains de personnes ignorantes et peu scrupuleuses. Il serait d'autre part regrettable que les grandes possibilités offertes par ces substances fassent oublier les règles élémentaires de l'hygiène qui gardent toujours toute leur valeur.

\section{CONCLUSION}

Les essais de conservation de la viande par l'auréomycine en milieu tropical, conduits à Dakar, sont exposés.

Les buts de l'expérimentation, le protocole et les résultats sont développés.
I a méthode la plus favorable de conservation, dans les conditions locales, 'semble être l'injection intra-veineuse d'auréomycine, deux heures avant l'abatlage des animaux pour la boucherie, à la dose d'un gramme d'antibiotique pour $100 \mathrm{~kg}$ de poids vif, complétée, après abattage, par une pulvérisation de la carcasse.

Nous pensons que le test le plus fidèle pour l'eppréciation de l'ćtat de conscrvation rclative de la viande est le dosage de l'azote basique volatil par la méthode de Conway. Ce fait opparaît clairement grâce à l'exploitation statistique des résultats.

Laboratoire central de l'Elevage " Georges Curasson" à Dalear. Directeur P. Mornet.

\section{BIBLIOGRAPHIE}

Andersen (A.-A.) et Michener (H.-D.). - Préservation of Foods with Antibiotics. I. The Complementary Action of Subtilin and Mild Heat. - Food Tech. 1950, 47, 188-189.

Anonymes. - Discussion autour des antibiotiques. - Rev. Cons. France, 1951, janvier, p. 51. Azote basique volatil total en inspection des viandes. - Encycl. vét. pér. 1947, p. 417. De la viande tendre avec les antibiotitiques. - Die Fleischwirtschaft 1956, 8, 231. Analyse in Bull. Inst. intern. Froid 1956, 36, 905.

Les antibiotiques peuvent-ils réduire le temps de stérilisation? - Rev. Cons. France $1950, \mathbf{n}^{\circ} 2$, p. 28.

Sur l'emploi des antibiotiques dans la conservation des denrées alimentaires. Rev. Cons. France 1950, no 6, p. 42-43.

Etat actuel des recherches sur la conser vation des aliments à l'aide des antibiotiques. - Revv. Cons. France, 1950, no 8, p. 29-34.

Bidault (C.). - Conservation de la viande et du poisson. - Baillière et fils Edit., Paris 1927.

Blanchard (L.), Nevot (A.), Pantaleon (J.) et PoIsson (J.). - Recherche sur le pH des viandes de boucherie. - Bull. Acad. vét. 1951, 24, 34-38.

DERRE (Y.). - Les souillures microbiennes des viandes. - La bactériémie d'abattage. Thèse Doctorat Vétérinaire, Alfort, 1952.

Dri guX (H.). - Le pH des viandes, sa mesure, ses indications. - Enclycl. vét. pér. 1944, p. $447-454$. 
Durresnoy (C.-H.). - Antibiotiques et conser vation des produits alimentaires d'origine animale. - Rev. Path. gén. 1957, 57, 175- 176.

Ginsberg (A.), Hill (E.-C.), Grieve (J.-M.). Oxytetracycline and its Use as a Meat Preservative in under-Developed Countries. Vet. Record 1957, 69, 983-993.

InGRAM (M.). - Fatigue musculaire, pH et prolifération bactérienne dans la viande. An. Inst. Past. 1948, 75, 139.

Ingram (M.), Barnes (E.) et ShewAN (I.). - Utilisation des antibiotiques pour la conservation de la viande et du poisson. - Food. Sci. 4bstr. (avril 1956), p. 121-136; analyse in Bull. Inst. intern. Froid. 1956, 36, 901.
LURo (P.). - La putréfaction en matière de viande fraîche d'animaux de boucherie. Thèse Doctorat vétérinaire Toulouse 1950.

NEVOT (A.). - Inspection bactériologique des viandes fraîches. Bull. Acad. vét. 1947, 20, 44.48 .

RAVINA (A.). - Une utilisation inattendue de la terramycine. - Presse méd. 1957, 65, 1068.

Thielilis (G.). - Nouveaux procédés de conservation des aliments. - Encycl. vét. pér. 1957, no $3,149-155$.

$V_{E L U}(M)$. - Les antibiotiques et la production agricole. Rev. Path. gén. 1957, 57, 1055 1093.

\section{SUMMARY}

\section{The Preservation of Meat in a Tropical Climate by the Addition of Aureomycin.}

The authors describe the trials made at Dakar on the preservation of meat by aureomycin. They detail the protocols of the experiments carried out and the results obtained. The method under local conditions which seems most favourable is the intravenous injection of aureomycin two hours before slaughter of butcher animals at the dosage rate of $1 \mathrm{~g}$ of antibiotic per $100 \mathrm{~kg}$ liveweight, followed after slaughter by spraying of the dressed carcass with a $25 \mathrm{cg} / 1000 \mathrm{ml}$ aqueous solution of aureomycin, using about 0.5 litre per carcass.

The authors are of the opinion that the most useful test of the preservation of the meat is the method of Conway to detect the quantity of volatile ammonia in comparison with an untreated carcass. The results are subject to a statistical analysis of a large number of tests.

\section{RESUMEN}

\section{La conservación de la carne en zonas tropicales, por medio de la aureomicina.}

Los autores exponen los ensayos de conservatión de las carne por la aureomicina, que han adelantado en Dakar. Explican el desarrollo, finalidades y resultados de éstas experiencias. El método que les parece más favorable, en las condiciones locales es la injección intravenosa de aureomicina, a la dosis de un gramo de antibiotico por $100 \mathrm{~kg}$ de peso vivo, dos horas antes del sacrificio de los animales, completada despues de la muerte por una pulverización de la carcasa con una solución de aureomicina a $25 \mathrm{cgm}$ par litro, a razón de un medio litro por carcasa.

Los autores piensan que el test más fiel para la aprcciación del cstado de conservación de la carne es la dosificatión de nitrógeno básico volatil por el método de Conway, según lo revelan las estadisticas sobre los resultados. 